\title{
FRACTAL DIMENSIONS AND RANDOM TRANSFORMATIONS
}

\author{
YURI KIFER
}

\begin{abstract}
I start with random base expansions of numbers from the interval $[0,1]$ and, more generally, vectors from $[0,1]^{d}$, which leads to random expanding transformations on the $d$-dimensional torus $\mathbb{T}^{d}$. As in the classical deterministic case of Besicovitch and Eggleston I find the Hausdorff dimension of random sets of numbers with given averages of occurrences of digits in these expansions, as well as of general closed sets "invariant" with respect to these random transformations, generalizing the corresponding deterministic result of Furstenberg. In place of the usual entropy which emerges (as explained in Billingsley's book) in the Besicovitch-Eggleston and Furstenberg cases, the relativised entropy of random expanding transformations comes into play in my setup. I also extend to the case of random transformations the Bowen-Ruelle formula for the Hausdorff dimension of repellers.
\end{abstract}

\section{INTRODUCTION}

Suppose we throw a fair die repeatedly and obtain a sequence of outcomes $m_{1}, m_{2}, \ldots, m_{i} \in\{1,2, \ldots, 6\}$. Then any $x \in[0,1]$ has a "random base" expansion

$$
x=\sum_{i=1}^{\infty} \frac{x_{i-1}}{m_{1} m_{2} \cdots m_{i}}, \text { where } x_{i} \in\left\{0,1, \ldots, m_{i+1}-1\right\} .
$$

It is not difficult to observe that with probability one for almost all $x$ with respect to the Lebesgue measure on $[0,1]$ the frequency of a digit $k \in\{0,1, \ldots, 5\}$ in this expansion will be equal to $\frac{1}{6} \sum_{k+1 \leq l \leq 6} 1 / l$. Thus the subsets of $[0,1]$ which have other frequencies of digits in the above expansion will have Lebesgue measure zero, and one may be interested in computing the Hausdorff dimension of such sets, which in the deterministic case was done many years ago in the classical papers $[\mathrm{Be}]$ and $[\mathrm{Eg}]$ of Besicovitch and Eggleston. More generally, we can simultaneously throw $d$ dice each time, write $d$ respective "random base" expansions, and study the dimensions of subsets of $[0,1]^{d}$ corresponding to different frequencies of vectors of $d$ digits in these expansions.

The general setup includes an ergodic measure preserving transformation $\theta$ of a probability space $(\Omega, P)$ and a $\mathbb{Z}_{+}^{d}$-valued random vector $m(\omega)=\left(m_{1}(\omega), \ldots\right.$, $\left.m_{d}(\omega)\right), \omega \in \Omega, m_{i}(\omega) \in \mathbb{Z}_{+}=\{1,2, \ldots\}$, such that for all $i=1, \ldots, d$,

$$
0<\int \log m_{i} d P=\int \log m_{1} d P<\infty .
$$

Received by the editors November 30, 1994 and, in revised form, June 16, 1995.

1991 Mathematics Subject Classification. Primary 28A78, Secondary 58F15, 28A80, 60F10.

Key words and phrases. Hausdorff dimension, random transformations, repellers.

Partially supported by the US-Israel Binational Science Foundation. 
For any $x=\left(x_{1}, \ldots, x_{d}\right) \in[0,1)^{d}$ one can write

$$
x=\sum_{j=0}^{\infty} x_{j}(\omega)\left(M\left(\theta^{j} \omega\right) \cdots M(\theta \omega) M(\omega)\right)^{-1},
$$

where $M(\omega)$ is the $d \times d$ diagonal matrix with the diagonal elements $m_{1}(\omega), \ldots, m_{d}(\omega)$ and $x_{j}(\omega)=\left(x_{1 j}(\omega), \ldots, x_{d j}(\omega)\right)$ are row vectors with $x_{i j}(\omega) \in\left\{0,1, \ldots, m_{i}\left(\theta^{j} \omega\right)-1\right\}$. This expansion is always possible since for $x_{i} \in[0,1)$ we can set $x_{i 0}(\omega)=\left[x_{i} m_{i}(\omega)\right]$, where [.] denotes the integer part, and

$$
x_{i j}(\omega)=\left[m_{i}(\omega) m_{i}(\theta \omega) \cdots m_{i}\left(\theta^{j} \omega\right)\left(x-\sum_{n=0}^{j-1} \frac{x_{i n}(\omega)}{m_{i}(\omega) \cdots m_{i}\left(\theta^{n} \omega\right)}\right)\right]
$$

after $x_{i 0}(\omega), \ldots, x_{i, j-1}(\omega)$ have aleady been defined. Thus zero tails are permitted but the tails $x_{i j}(\omega)=m_{i}\left(\theta^{j} \omega\right)-1$, for all $j \geq n$ are not. For any $k, l \in \mathbb{Z}_{+}^{d}$ set

$$
\begin{aligned}
N_{k l}^{\omega}(x, n)=\mid\left\{j \geq 0, j<n: m\left(\theta^{j} \omega\right)\right. & \left.=k, x_{j}(\omega)=l-\mathbf{1}\right\} \mid, \\
N_{l}^{\omega}(x, n) & =\sum_{k \in \mathbb{Z}_{+}^{d}} N_{k l}^{\omega}(x, n),
\end{aligned}
$$

where $\mathbf{1}=(1, \ldots, 1) \in \mathbb{Z}_{+}^{d}$ and $|\{\cdot\}|$ denotes the number of elements in a set $\{\cdot\}$. Let $r=\left(r_{k}, k \in \mathbb{Z}_{+}^{d}\right)$ be an infinite probability vector and $A=\left(a_{k l}, k, l \in \mathbb{Z}_{+}^{d}\right)$ be an infinite probability matrix such that $a_{k l}=0$ unless $l \leq k$, where $l=\left(l_{1}, \ldots, l_{d}\right) \leq$ $k=\left(k_{1}, \ldots, k_{d}\right)$ means that $l_{i} \leq k_{i}, \forall i=1, \ldots, d$. I shall compute for $P$-almost all (a.a.) $\omega \in \Omega$ the Hausdorff dimensions (which will be denoted by $H D$ ) of the sets

$$
U_{r}^{\omega}=\left\{x \in[0,1)^{d}: \lim _{n \rightarrow \infty} \frac{1}{n} N_{l}^{\omega}(x, n)=r_{l} \text { for all } l \in \mathbb{Z}_{+}^{d}\right\}
$$

and

$$
V_{A}^{\omega}=\left\{x \in[0,1)^{d}: \lim _{n \rightarrow \infty} \frac{1}{n} N_{k l}^{\omega}(x, n)=q_{k} a_{k l} \text { for all } k, l \in \mathbb{Z}_{+}^{d}\right\}
$$

where $q_{k}=P\{m=k\}$. By ergodicity of $\theta$ it follows that $H D\left(U_{r}^{\omega}\right)$ and $H D\left(V_{A}^{\omega}\right)$ are the same for $P$-a.a. $\omega$. I obtain these Hausdorff dimensions in Theorem 2.1 of the next section. If $d=1$ and $m$ is constant with probability one, then we get the results from $[\mathrm{Be}]$ and $[\mathrm{Eg}]$. B. Mandelbrot pointed out to me the paper of Peyrière [Pey], who computed the Hausdorff dimension of sets similar to $V_{A}^{\omega}$ with $d=1$, which correspond to expansions of numbers from $[0,1]$ with respect to nonrandom sequences of positive integers satisfying certain conditions which, in general, do not hold true with probability one for the sequence $m_{1}(\omega), m_{1}(\theta \omega), m_{1}\left(\theta^{2} \omega\right), \ldots$ assuming only (1.1).

Define the transformations $f(\omega):[0,1)^{d} \rightarrow[0,1)^{d}$ and $\tau:[0,1)^{d} \times \Omega \rightarrow[0,1)^{d} \times \Omega$ by the formulas

$$
(f(\omega) x)_{i}=m_{i}(\omega) x_{i}-\left[m_{i}(\omega) x_{i}\right] \quad \forall i=1, \ldots, d \text { and } x=\left(x_{1}, \ldots, x_{d}\right) \in[0,1)^{d}
$$


and

$$
\tau(x, \omega)=(f(\omega) x, \theta \omega) .
$$

It is convenient to identify $[0,1)^{d}$ with the $d$-dimensional torus $\mathbb{T}^{d}$, and from now on I assume that the expansion (1.2) is given for points of $\mathbb{T}^{d}$. Thus $f(\omega)$ becomes a random smooth expanding transformation of $\mathbb{T}^{d}$ which I use frequently in this paper.

For any $x \in \mathbb{T}^{d}$ and $\omega \in \Omega$ set $\phi(x, \omega)=x_{0}(\omega) \in \mathbb{Z}_{+}^{d}$. Then $x_{1}(\omega)=$ $(f(\omega) x)_{0}(\theta \omega)=(\phi \circ \tau)(x, \omega)$, and so by induction, assuming that $\left(\phi \circ \tau^{i-1}\right)(x, \omega)=$ $x_{i-1}(\omega)$, I obtain

$$
\left(\phi \circ \tau^{i}\right)(x, \omega)=\left(\phi \circ \tau^{i-1}\right)(f(\omega) x, \theta \omega)=(f(\omega) x)_{i-1}(\theta \omega)=x_{i}(\omega) .
$$

This connection between the expansion (1.2) and the skew product transformation $\tau$ enables me to compute $H D\left(U_{r}^{\omega}\right)$ and $H D\left(V_{A}^{\omega}\right)$ by modifying Billingsley's entropy approach to the results from [Be] and [Eg] given in [Bi1]-[Bi3]. Employing the same machinery I obtain in Section 3 Hausdorff dimensions of more general sets, relax the condition (1.1), and also derive almost sure large deviations bounds for $\frac{1}{n} N_{k l}^{\omega}(x, n)$ and $\frac{1}{n} N_{l}^{\omega}(x, n)$.

Let $K^{\omega}, \omega \in \Omega$, be a measurable family of compact subsets of $\mathbb{T}^{d}$ such that

$$
f(\omega) K^{\omega}=K^{\theta \omega} .
$$

One obtains such sets, for instance, by considering all $x \in \mathbb{T}^{d}$ whose expansion (1.2) does not contain certain prescribed "vector-digits" which can be called random Cantor sets. Generalizing Furstenberg's deterministic formula from [Fu], I shall show in Section 4 that for compact $K^{\omega}$ satisfying (1.10) with probability one,

$$
H D\left(K^{\omega}\right)=\frac{h_{\mathrm{top}}^{(r)}(\tau, K)}{\int m_{1} d P}
$$

where $h_{\text {top }}^{(r)}(\tau, K)$ is the relativized topological entropy of $\tau$ restricted to the invariant set $K=\left\{(x, \omega): x \in K^{\omega}\right\}$. Note that the sets $U_{r}^{\omega}$ and $V_{A}^{\omega}$ also satisfy (1.10), but they are usually everywhere dense in $\mathbb{T}^{d}$, and so they should be treated differently. The Hausdorff and Minkowski dimensions of random Cantor sets $K^{\omega}$ will be computed also in the case when the integrals in (1.1) are not the same for different $i$ 's; this yields random versions of results from [Mc] and [KP2]. Finally, I shall obtain the Hausdorff dimension of general "random invariant repellers" for random conformal maps, which generalizes the corresponding deterministic Bowen-Ruelle formula (see $[\mathrm{Ru}]$ ). In this case the relativised topological pressure comes into the picture in place of the relativized topological entropy above. I also define random iterated function systems and explain that some of my models can be studied also via this setup.

\section{RANDOM BASE EXPANSIONS}

By (1.4)-(1.6) and (1.9) I can write

$$
U_{r}^{\omega}=\left\{x \in \mathbb{T}^{d}: \lim _{n \rightarrow \infty} \frac{1}{n} \sum_{i=0}^{n-1} \chi_{l-1}\left(\phi \circ \tau^{i}(x, \omega)\right)=r_{l} \forall l \in \mathbb{Z}^{d}\right\}
$$


where $\chi_{l}(k)=1$ if $l=k$ and $\chi_{l}(k)=0$ otherwise, and

$$
\begin{aligned}
V_{A}^{\omega}=\left\{x \in \mathbb{T}^{d}: \lim _{n \rightarrow \infty} \frac{1}{n} \sum_{i=0}^{n-1} \chi_{k}\left(m \circ \theta^{i}(\omega)\right) \chi_{l-1}\left(\phi \circ \tau^{i}(x, \omega)\right)\right. & \\
& \left.=q_{k} a_{k l} \quad \text { for all } l, k \in \mathbb{Z}_{+}^{d}, l \leq k\right\} .
\end{aligned}
$$

Therefore, with probability one,

$$
\begin{aligned}
f(\omega) V_{A}^{\omega}=\left\{y \in \mathbb{T}^{d}: \lim _{n \rightarrow \infty} \frac{1}{n} \sum_{i=0}^{n-2} \chi_{k}\left(m \circ \theta^{i}(\theta \omega)\right) \chi_{l-1}\left(\phi \circ \tau^{i}(y, \theta \omega)\right)\right. & \\
& \left.=q_{k} a_{k l} \quad \text { for all } l, k \in \mathbb{Z}_{+}^{d}, l \leq k\right\}=V_{A}^{\theta \omega}
\end{aligned}
$$

and similarly,

$$
f(\omega) U_{r}^{\omega}=U_{r}^{\theta \omega}
$$

Thus, clearly,

$$
H D\left(U_{r}^{\omega}\right)=H D\left(U_{r}^{\theta \omega}\right) \quad \text { and } \quad H D\left(V_{A}^{\omega}\right)=H D\left(V_{A}^{\theta \omega}\right),
$$

and since $\theta$ is ergodic and $H D\left(U_{r}^{\omega}\right), H D\left(V_{A}^{\omega}\right)$ depend measurably on $\omega$, I conclude that with probability one,

$$
H D\left(U_{r}^{\omega}\right)=\text { const and } H D\left(V_{A}^{\omega}\right)=\text { const. }
$$

The main result of this section is the following

2.1. Theorem. With probability one,

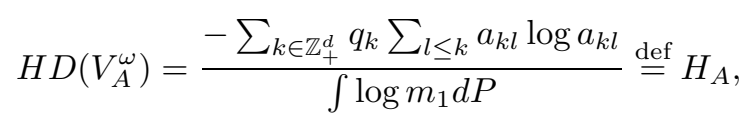

and so $H D\left(V_{A}^{\omega}\right)=d$ if and only if $a_{k l}=\prod_{i=1}^{d} k_{i}^{-1}$ for all $l \leq k$ and any $k=$ $\left(k_{1}, \ldots, k_{d}\right) \in \mathbb{Z}_{+}^{d}$ such that $q_{k} \neq 0$. In the last case with probability one $V_{A}^{\omega}$ also has Lebesgue measure one. The sets $U_{r}^{\omega}$ have Lebesgue measure one for P-a.a. $\omega$ if and only if $r_{l}=\sum_{k \geq l} q_{k} \prod_{i=1}^{d} k_{i}^{-1}$ for all $l \in Z_{+}^{d}$. Furthermore, for $P$-a.a. $\omega$,

$$
H D\left(U_{r}^{\omega}\right)=\sup _{A \in \mathcal{A}_{q r}} H_{A} \stackrel{\text { def }}{=} H
$$

where the supremum in (2.8) is taken over the set $\mathcal{A}_{q r}$ of all infinite probability matrices $A=\left(a_{k l}\right)$ such that $a_{k l}=0$ unless $l \leq k$ and $q A=r$ with $q$ and $r$ considered 
as row vectors (i.e. $\sum_{k \in \mathbb{Z}_{+}^{d}} q_{k} a_{k l}=r_{l}, \forall l \in \mathbb{Z}_{+}^{d}$ ). The set $\mathcal{A}_{q r}$ is nonempty if and only if

$$
\sum_{l \in F} q_{l} \geq \sum_{l \in F} r_{l}
$$

for any $F \in \mathcal{F}$, where $\mathcal{F}$ is the collection of all filters in $\mathbb{Z}_{+}^{d}$, i.e. the subsets $F \subset \mathbb{Z}^{d}$ such that if $l \in F$ and $l \leq k$, then $k \in F$. If (2.9) does not hold true for some $F \in \mathcal{F}$, then with probability one $U_{r}^{\omega}$ is empty.

Proof. By the definition (1.4),

$$
\sum_{l \in F} N_{l}^{\omega}(x, n)=\sum_{l \in F} \sum_{k \in \mathbb{Z}_{+}^{d}} N_{k l}^{\omega}(x, n)=\sum_{k \in F} \sum_{l \in F} N_{k l}^{\omega}(x, n) \leq \sum_{k \in F} M_{k}^{\omega}(n),
$$

where

$$
M_{k}^{\omega}(n)=\left|I_{k}^{\omega}(n)\right| \quad \text { and } \quad I_{k}^{\omega}(n)=\left\{j \geq 0: m\left(\theta^{j} \omega\right)=k, j<n\right\},
$$

and so by Birkhoff's ergodic theorem, with probability one,

$$
\limsup _{n \rightarrow \infty} \frac{1}{n} \sum_{l \in F} N_{l}^{\omega}(x, n) \leq \sum_{k \in F} q_{k}
$$

Hence, if (2.9) does not hold true then $U_{r}^{\omega}$ is empty with probability one.

For each $\kappa>0$ and a set $M \subset \mathbb{T}^{d}$ put

$$
\ell_{\kappa}(M, \rho)=\inf \sum_{i}\left|v_{i}\right|^{\kappa}
$$

where the infinum is taken over all covers of $M$ by cubes $v_{i}$ with sides of length not exceeding $\rho$, where $\left|v_{i}\right|$ denotes the volume of $v_{i}$. Let $\alpha_{0}=\left(\alpha_{10}, \ldots, \alpha_{d 0}\right), \ldots, \alpha_{n-1}$ $=\left(\alpha_{1, n-1}, \ldots, \alpha_{d, n-1}\right)$ be some integer vectors such that $0 \leq \alpha_{i j}<m_{i}\left(\theta^{j} \omega\right)$. Set

$$
C_{\alpha_{0}, \ldots, \alpha_{n-1}}^{\omega}=\left\{x \in \mathbb{T}^{d}: x_{j}(\omega)=\alpha_{j}, \forall j=0,1, \ldots, n-1\right\},
$$

which I shall call an $(\omega, n)$-cylinder or just an $\omega$-cylinder if $n$ is not fixed. Note that each such cylinder is a rectangular parallelepiped with the volume

$$
\left|C_{a_{0}, \ldots, a_{n-1}}^{\omega}\right|=\left(\prod_{i=1}^{d}\left(\prod_{j=0}^{n-1} m_{i}\left(\theta^{j} \omega\right)\right)\right)^{-1}
$$

Set also

$$
\lambda_{\kappa}^{\omega}(M, \rho)=\inf \sum_{i}\left|C_{i}\right|^{\kappa}
$$

where the infinum is taken over all covers of $M \subset \mathbb{T}^{d}$ by $\omega$-cylinders $C_{i}$ whose maximal side does not exceed $\rho$, where $\left|C_{i}\right|$ is the volume of $C_{i}$. The following result is a generalization of an argument from [Bi 2]. 
2.2. Lemma. For any $\varepsilon>0$ and $P$-a.a. $\omega$ there exists $K_{\varepsilon}(\omega)>0$ such that for any set $M \subset \mathbb{T}^{d}, \rho>0$, and $\kappa>\varepsilon$,

$$
K_{\varepsilon}^{-1}(\omega) \ell_{\kappa+\varepsilon}(M, \rho) \leq \lambda_{\kappa}^{\omega}(M, \rho) \leq K_{\varepsilon}(\omega) \ell_{\kappa-\varepsilon}(M, \rho) .
$$

Proof. Take a cube $u \subset \mathbb{Z}^{d}$ and let $n$ be minimal such that $u \supset C_{\alpha_{0}, \ldots, \alpha_{n-1}}^{\omega}$ (i.e. $u$ does not contain any $(\omega, n-1)$-cylinder $\left.C_{\beta_{0}, \ldots, \beta_{n-2}}^{\omega}\right)$. Then it is easy to see that $u$ is covered by no more than $2^{d} \prod_{i=1}^{d} m_{i}\left(\theta^{n-1} \omega\right)(\omega, n)$-cylinders (whose maximal side obviously does not exceed the side of $u$ ). By (2.13) I derive that

$$
\left|C_{\alpha_{0}, \ldots, \alpha_{n-1}}^{\omega}\right|^{\kappa} \leq|u|^{\kappa} \leq\left(\left(2^{d} \prod_{i=1}^{d} m_{i}\left(\theta^{n-1} \omega\right)\right)^{\kappa}\left|C_{\alpha_{0}, \ldots, \alpha_{n-1}}^{\omega}\right|^{\kappa}\right) \leq K_{\varepsilon}^{(n)}(\omega)|u|^{\kappa-\varepsilon}
$$

where

$$
K_{\varepsilon}^{(n)}=2^{d(\kappa-\varepsilon)}\left(\prod_{i=1}^{d} m_{i}\left(\theta^{n-1} \omega\right)\right)^{\kappa}\left(\prod_{i=1}^{d}\left(\prod_{j=0}^{n-2} m_{i}\left(\theta^{j} \omega\right)\right)\right)^{-1} .
$$

Next, observe that any cylinder $C_{\alpha_{0}, \ldots, \alpha_{n-1}}^{\omega}$ contains a cube $u$ with side equal to the minimal side of $C_{\alpha_{0}, \ldots, \alpha_{n-1}}^{\omega}$, and so each such cylinder can be covered by no more than

$$
k_{n}(\omega)=\left(1+\left[\max _{i, j}\left(\prod_{a=0}^{n-1} \frac{m_{i}\left(\theta^{a} \omega\right)}{m_{j}\left(\theta^{a} \omega\right)}\right)\right]\right)^{d}
$$

of such cubes $u$, where $[\cdot]$ denotes the integer part. Then

$$
k_{n}(\omega)|u|^{\kappa+\varepsilon} \leq L_{\varepsilon}^{(n)}(\omega)\left|C_{\alpha_{0}, \ldots, \alpha_{n-1}}^{\omega}\right|^{\kappa},
$$

where

$$
L_{\varepsilon}^{(n)}(\omega)=k_{n}(\omega)|u|^{\varepsilon}=k_{n}(\omega) \min _{i}\left(\prod_{j=0}^{n-1} m_{i}\left(\theta^{j} \omega\right)\right)^{-1} .
$$

Observe that, by (2.1) and the Birkhoff ergodic theorem, with probability one for each $i=1, \ldots, d$,

$$
\lim _{n \rightarrow \infty} \frac{1}{n} \sum_{j=0}^{n-1} \log m_{i}\left(\theta^{j} \omega\right)=\int \log m_{1} d P>0,
$$

and by the ergodic theorem, with probability one,

$$
\lim _{n \rightarrow \infty} \frac{1}{n} \log m_{i}\left(\theta^{n} \omega\right)=\lim _{n \rightarrow \infty} \frac{1}{n}\left(\sum_{j=0}^{n} \log m_{i}\left(\theta^{j} \omega\right)-\sum_{j=0}^{n-1} \log m_{i}\left(\theta^{j} \omega\right)\right)=0 .
$$

It follows that with probability one

$$
\lim _{n \rightarrow \infty} K_{\varepsilon}^{(n)}=\lim _{n \rightarrow \infty} L_{\varepsilon}^{(n)}=0 .
$$


Then with probability one $K_{\varepsilon}=\sup _{n>2} \max \left(K_{\varepsilon}^{(n)}, L_{\varepsilon}^{(n)}\right)<\infty$, and I derive (2.15) with such $K_{\varepsilon}$ in view of (2.16) and (2.17).

Next, similarly to [Bi1] and [Bi2], for any nonatomic probability measure $\mu$ on $\mathbb{T}^{d}$ set

$$
\mu_{\kappa}^{\omega}(M, \rho)=\inf \sum_{i} \mu\left(C_{i}\right)^{\kappa}
$$

where the infinum is taken over all covers by $\omega$-cylinders $C_{i}$ such that $\mu\left(C_{i}\right)<\rho$. Note that if $\mu$ is the Lebesgue measure on $\mathbb{T}^{d}$, then $\mu_{\kappa}^{\omega}(M, \rho)=\lambda_{\kappa}^{\omega}(M, \rho)$. By the monotonicity of $\ell_{\kappa}(M, \rho), \lambda_{\kappa}^{\omega}(M, \rho)$, and $\mu_{\kappa}^{\omega}(M, \rho)$ in $\rho$ there exist the limits

$$
\begin{aligned}
\ell_{\kappa}(M) & =\lim _{\rho \rightarrow 0} \ell_{\kappa}(M, \rho), \quad \lambda_{\kappa}^{\omega}(M)=\lim _{\rho \rightarrow 0} \lambda_{\kappa}^{\omega}(M, \rho), \\
\mu_{\kappa}^{\omega}(M) & =\lim _{\rho \rightarrow 0} \mu_{\kappa}^{\omega}(M, \rho) .
\end{aligned}
$$

By Lemma 2.2 , for $P$-a.a. $\omega$,

$$
H D(M)=\kappa_{0} \quad \text { iff } \quad \lambda_{\kappa}^{\omega}(M)=\infty, \forall \kappa<\kappa_{0} \text { and } \lambda_{\kappa}^{\omega}(M)=0, \forall \kappa>\kappa_{0} .
$$

Set

$$
H D_{\mu}^{\omega}(M)=\inf \left\{\kappa>0: \mu_{\kappa}^{\omega}(M)=0\right\}
$$

then it is easy to check (see [Bi1]) that $\mu_{\kappa}^{\omega}(M)=\infty$ for all $\kappa<H D_{\mu}^{\omega}(M)$. By Lemma 2.2,

$$
H D_{\mathrm{Leb}}^{\omega}(M)=\inf \left\{\kappa>0: \lambda_{\kappa}^{\omega}(M)=0\right\}=d^{-1} H D(M),
$$

where Leb denotes the Lebesgue measure on $\mathbb{T}^{d}$. For $x \in \mathbb{T}^{d}$ denote $C_{n}(x)=$ $C_{x_{0}(\omega), \ldots, x_{n-1}(\omega)}^{\omega}$. Let $\mu$ and $\nu$ be nonatomic probability measures on $\mathbb{T}^{d}$ and $\delta=\delta^{\omega}$ a nonnegative random variable. Set

$$
\underline{X}_{\mu, \nu}^{\omega}(\delta)=\left\{x: \delta^{\omega} \leq \liminf _{n \rightarrow \infty} \frac{\log \nu\left(C_{n}^{\omega}(x)\right)}{\log \mu\left(C_{n}^{\omega}(x)\right)}\right\}
$$

and

$$
\bar{X}_{\mu, \nu}^{\omega}(\delta)=\left\{x: \limsup _{n \rightarrow \infty} \frac{\log \nu\left(C_{n}^{\omega}(x)\right)}{\log \mu\left(C_{n}^{\omega}(x)\right)} \leq \delta^{\omega}\right\}
$$

with the convention that $\log a / \log 0=\log 1 / \log 0=0, \log 0 / \log a=\log a / \log 1$ $=\log 0 / \log 1=\infty$ and $\log 0 / \log 0=\log 1 / \log 1=1$ for any $a \in(0,1)$. The following is a version of Theorems 2.2 and 2.3 from [Bi1,II] (see also Theorem 14.1 from [Bi2], Section 5 in [Bi3], and Proposition 2.1 in [Yo]).

2.3. Proposition. Let $M, N$ be subsets of $\mathbb{T}^{d}$ and $\underline{\delta}, \bar{\delta}$ nonnegative random variables. Then for all $\omega \in \Omega$,

$$
\text { if } M \subset \underline{X}_{\mu, \nu}^{\omega}(\underline{\delta}) \text {, then } H D_{\mu}^{\omega}(M) \geq \underline{\delta}^{\omega} H D_{\nu}^{\omega}(M)
$$

and

$$
\text { if } N \subset \bar{X}_{\mu, \nu}^{\omega}(\bar{\delta}) \text {, then } H D_{\mu}^{\omega}(N) \leq \bar{\delta}^{\omega} H D_{\nu}^{\omega}(N) \text {. }
$$


Moreover,

$$
H D_{\mu}^{\omega}\left\{x: \liminf _{n \rightarrow \infty} \frac{\log \nu\left(C_{n}^{\omega}(x)\right)}{\log \mu\left(C_{n}^{\omega}(x)\right)} \leq \bar{\delta}^{\omega}\right\} \leq \bar{\delta}^{\omega}
$$

Proof. For each $\omega \in \Omega$ I can define the stochastic process $y_{n}^{\omega}(x)=x_{n}(\omega)$ on the probability space $\left(\mathbb{T}^{d}, \mu\right)$, and this is precisely the setup of Theorems 2.2 and 2.3 from [Bi1,II] which imply Proposition 2.3 above.

Next, I shall apply Proposition 2.3 with $\mu=$ Leb, taking into account that, by $(2.13)$

$$
\operatorname{Leb}\left(C_{n}^{\omega}(x)\right)=\left(\prod_{i=1}^{d}\left(\prod_{j=0}^{n-1} m_{i}\left(\theta^{j} \omega\right)\right)\right)^{-1},
$$

and so, by Birkhoff's ergodic theorem,

$$
\begin{aligned}
Y_{\nu}^{\omega}(\gamma) & \stackrel{\text { def }}{=}\left\{x: \lim _{n \rightarrow \infty}\left(-\frac{1}{n} \log \nu\left(C_{n}^{\omega}(x)\right)\right)=\gamma\right\} \\
& =\left\{x: \lim _{n \rightarrow \infty}\left(\frac{\frac{1}{n} \log \nu\left(C_{n}^{\omega}(x)\right)}{\frac{1}{n} \log \operatorname{Leb}\left(C_{n}^{\omega}(x)\right)}\right)=\frac{\gamma}{d \int \log m_{1} d P}\right\} .
\end{aligned}
$$

Then, by (2.22) and Proposition 2.3,

$$
\text { if } M \subset Y_{\nu}^{\omega}(\gamma) \text {, then } H D(M)=\frac{\gamma}{\int \log m_{1} d P} H D_{\nu}^{\omega}(M) \text {. }
$$

By (2.18) for each $x$ and $P$-a.a. $\omega, C_{n}^{\omega}(x) \downarrow\{x\}$ as $n \uparrow \infty$, and so for any nonatomic measure $\nu$ with probability one $\nu\left(C_{n}^{\omega}(x)\right) \rightarrow 0$ as $n \rightarrow \infty$. It is easy to see (cf. [Bi1,2]) that for such $\nu$ if $\nu(M)>0$ then $H D_{\nu}^{\omega}(M)=1$ with probability one. Thus by $(2.27)$, for $P$-a.a. $\omega$,

$$
\text { if } M \subset Y_{\nu}^{\omega}(\gamma) \text { and } \nu(M)>0 \text {, then } H D(M)=\frac{\gamma}{\int \log m_{1} d P} .
$$

Next, in order to obtain (2.7) via (2.28) I should construct an appropriate family of measures $\nu^{\omega}, \omega \in \Omega$. Consider a measurable family $p^{\omega}, \omega \in \Omega$, of infinite probability vectors $p^{\omega}=\left(p_{l}^{\omega} ; l \in \mathbb{Z}_{+}^{d}\right)$ such that $\sum_{l \leq m(\omega)} p_{l}^{\omega}=1$. Define

$$
\nu_{p}^{\omega}\left(C_{\alpha_{0}, \ldots, \alpha_{n-1}}^{\omega}\right)=\prod_{j=0}^{n-1} p_{\alpha_{j}+\mathbf{1}}^{\theta^{j} \omega} .
$$

Since for $P$-a.a. $\omega$ the $\omega$-cylinders generate the whole Borel $\sigma$-algebra on $\mathbb{T}^{d}$, then by the Kolmogorov extension theorem for $P$-a.a. $\omega$ there exists a unique (and so measurably depending on $\omega \in \Omega$ ) probability measure $\nu_{p}^{\omega}$ on $\mathbb{T}^{d}$ satisfying (2.29). Observe that if $p_{l}^{\omega}=\left(m_{1}(\omega) \cdots m_{d}(\omega)\right)^{-1}$ for all $l \leq m(\omega), l \in \mathbb{Z}_{+}^{d}$ and $\omega \in \Omega$, then 
$\nu_{p}^{\omega}=$ Leb for all $\omega$, since then $\nu_{p}\left(C_{\alpha_{0}, \ldots, \alpha_{n-1}}^{\omega}\right)$ equals the right hand side of (2.13). Next,

$$
\begin{aligned}
& \left(f(\omega) \nu_{p}^{\omega}\right)\left(C_{\alpha_{0}, \ldots, \alpha_{n-1}}^{\theta \omega}\right)=\nu_{p}^{\omega}\left(f^{-1}(\omega) C_{\alpha_{0}, \ldots, \alpha_{n-1}}^{\theta \omega}\right)=\sum_{\beta \leq m(\omega)-\mathbf{1}} \nu_{p}^{\omega}\left(C_{\beta, \alpha_{0}, \ldots, \alpha_{n-1}}^{\omega}\right) \\
& =\sum_{\beta \leq m(\omega)-\mathbf{1}} p_{\beta+\mathbf{1}}^{\omega} \prod_{j=0}^{n-1} p_{\alpha_{j}+\mathbf{1}}^{\theta^{j+1} \omega}=\prod_{j=0}^{n-1} p_{\alpha_{j}+\mathbf{1}}^{\theta^{j+1} \omega}=\nu_{p}^{\theta \omega}\left(C_{\alpha_{0}, \ldots, \alpha_{n-1}}^{\theta \omega}\right) .
\end{aligned}
$$

Since this is true for any $\omega$-cylinder, I derive that for $P$-a.a. $\omega$,

$$
f(\omega) \nu_{p}^{\omega}=\nu_{p}^{\theta \omega}
$$

Define the probability measure $\nu_{p}$ on $\mathbb{T}^{d} \times \Omega$ by $\nu_{p}(W)=\int \nu_{p}^{\omega}\left(W^{\omega}\right) d P(\omega)$, where $W^{\omega}=\left\{x \in \mathbb{T}^{d}:(x, \omega) \in W\right\}$ and $W \subset \mathbb{T}^{d} \times \Omega$. Then (see [Ki2]) (2.30) implies that $\nu_{p}$ is invariant with respect to the skew product transformation $\tau$ given by (1.8). Moreover, it turns out that $\nu_{p}$ is ergodic (see $[\mathrm{KK}]$ and Remark 2.8 below) since it is the unique equilibrium state corresponding to the function $\psi(x, \omega)=\log p_{x_{0}(\omega)+1}^{\omega}$ (such $\nu_{p}$ is naturally called "a random Bernoulli measure"). This argument requires an additional justification in the case when some of the $p_{l}^{\omega}$ are equal to 0 , and so the function $\psi$ may take the value $-\infty$. Namely, I construct the measures $\nu_{p}^{\omega}$ only on the subset of $x$ 's such that $x_{i}(\omega) \in\left\{0,1, \ldots, m\left(\theta^{i} \omega\right)\right\}$ and $p_{x_{i}(\omega)+1}^{\theta^{i} \omega}>0$. In the symbolic representation this corresponds to the sequences $\left(\alpha_{0}, \alpha_{1}, \ldots\right)$ with $p_{\alpha_{i}}^{\theta^{i} \omega}>0$ which determines a random subshift of finite type (see [BG] and [KK]). Again, the corresponding measure $\nu_{p}$ will be ergodic (see $[\mathrm{KK}]$ ). The measure $\nu_{p}^{\omega}$ will be nonatomic except for the special case when all the $p_{l}^{\omega}$ equal zero or one, only. Observe also that except for the last case, with probability one,

$$
\nu_{p}^{\omega}\left(\bigcup_{n ; \alpha_{0}, \ldots, \alpha_{n-1}} \partial C_{\alpha_{0}, \ldots, \alpha_{n-1}}^{\omega}\right)=0
$$

which is a partial case of the general statement saying that the boundaries of random Markov partitions for random expanding transformations have zero measure for any Gibbs measure (equilibrium state) constructed by a function $\psi(x, \omega)$ Hölder continuous in $x$ (see $[\mathrm{KK}]$ ).

Now let the probability vectors $p_{A}^{\omega}=\left(p_{l}^{\omega}, l \in \mathbb{Z}_{+}^{d}\right)$ be constructed by the matrix $A$ appearing in (1.6) so that $p_{l}^{\omega}=a_{k l}$ for $\omega \in\{\omega: m(\omega)=k\}, k \in \mathbb{Z}_{+}^{d}$. Assume first that $0<a_{k l}<1$ for all $l \leq k$ and $k \in \mathbb{Z}_{+}^{d}$. Then by (1.4),

$$
-\frac{1}{n} \log \nu_{p_{A}}^{\omega}\left(C_{n}^{\omega}(x)\right)=-\frac{1}{n} \sum_{j=0}^{n-1} \log p_{x_{j}(\omega)+1}^{\theta^{j} \omega}=-\frac{1}{n} \sum_{k \in \mathbb{Z}_{+}^{d}} \sum_{l \leq k} N_{k l}^{\omega}(x, n) \log a_{k l},
$$

provided $\nu_{p_{A}}^{\omega}\left(C_{n}^{\omega}(x)\right) \neq 0$, and so, by the definition (1.6),

$$
V_{A}^{\omega} \subset\left\{x: \lim _{n \rightarrow \infty}\left(-\frac{1}{n} \log \nu_{p_{A}}^{\omega}\left(C_{n}^{\omega}(x)\right)\right)=-\sum_{k \in \mathbb{Z}_{+}^{d}} q_{k} \sum_{l \leq k} a_{k l} \log a_{k l}\right\} .
$$


As I observed above, $\nu_{p_{A}}$ is an ergodic invariant measure of the skew product transformation $\tau$, and so, by Birkhoff's ergodic theorem for $\nu_{p_{A}}$-a.a. $(x, \omega)$,

$$
\begin{aligned}
& \lim _{n \rightarrow \infty} \frac{1}{n} \sum_{j=0}^{n-1} \chi_{k}\left(m \circ \theta^{j}(\omega)\right) \chi_{l-1}\left(\phi \circ \tau^{j}(x, \omega)\right) \\
& =\int \chi_{k}(m(\omega))\left(\int \chi_{l-1}\left(x_{0}(\omega)\right) d \nu_{p_{A}}^{\omega}(x)\right) d P(\omega)=q_{k} a_{k l} .
\end{aligned}
$$

By the Fubini theorem I conclude from (2.2) and (2.32) that $\nu_{p_{A}}^{\omega}\left(V_{A}^{\omega}\right)=1$ for $P$-a.a. $\omega$. This together with (2.31) enables me to apply (2.28), which yields (2.7). Observe that the function $-t \log t$ is strictly convex on $[0,1]$, and so $-\sum_{l \leq k} a_{k l} \log a_{k l}$ $\leq \log \prod_{i=1}^{d} k_{i}$ if $k=\left(k_{1}, \ldots, k_{d}\right) \in \mathbb{Z}_{+}^{d}$, with equality if and only if $a_{k l}=\left(\prod_{i=1}^{k} k_{i}\right)^{-1}$ for all $l \leq k, l \in \mathbb{Z}_{+}^{d}$. For such $A$ one has $\nu_{p_{A}}^{\omega}=$ Leb $P$-a.s., and $\operatorname{so} \operatorname{Leb}\left(V_{A}^{\omega}\right)=1$ $P$-a.s. It follows also by $(1.4),(2.1)$, and $(2.32)$ that for Leb $P$ - a.a. $(x, \omega)$,

$$
\lim _{n \rightarrow \infty} \frac{1}{n} N_{l}^{\omega}(x, n)=\sum_{k \geq l}\left(\prod_{i=1}^{d} k_{i}\right)^{-1} q_{k},
$$

and so $\operatorname{Leb}\left(U_{r}^{\omega}\right)=1$ with probability one if and only if $r_{l}=\sum_{k \geq l}\left(\prod_{i=1}^{d} k_{i}\right)^{-1} q_{k}$, for all $l \in \mathbb{Z}_{+}^{d}$. If some $a_{k l}$ equal 0 for $k$ with $q_{k}=0$, I just redefine $a_{k l}$ to make them positive without any influence on the final result. If $q_{k}>0$ and some $a_{k l}=0$, then the measure $\nu_{p_{A}}^{\omega}$ is still supported by those $x$ for which $p_{x_{i}(\omega)+1}^{\theta^{i} \omega}>0$, i.e. the set $\tilde{V}_{A}^{\omega}$ of such $x$ 's has $\nu_{p_{A}}^{\omega}$-measure equal to 1 . In general, $\tilde{V}_{A}^{\omega}$ is a proper subset of $V_{A}^{\omega}$, and so I obtain only the lower bound $H_{A}=H D\left(\tilde{V}_{A}^{\omega}\right) \leq H D\left(V_{A}^{\omega}\right)$. In order to obtain the upper bound in this case one can employ either direct combinatorial estimates as (2.38)-(2.39) below estimating the number of cylinder sets intersecting $V_{A}$, which resemble Eggleston's original estimates from $[\mathrm{Eg}]$, or proceed by an approximation argument described in Remark 2.5 below. Observe that even in the deterministic case, if some of the prescribed frequencies are zero the proof of the upper bound using (2.24) (or versions of it) does not work, and one should employ either combinatorial upper bound estimates from $[\mathrm{Eg}]$ or the argument from Remark 2.5 below. This point was overlooked in [Bi1-3] and in [Fa], and, it seems, nobody noticed it for more than 30 years since this proof first appeared.

Next, I shall derive (2.8). First, observe that by (1.4) if $A=\left(a_{k l}\right) \in \mathcal{A}_{q r}$ and $\lim _{n \rightarrow \infty} \frac{1}{n} N_{k l}^{\omega}(x, n)=q_{k} a_{k l}$ for all $l \leq k$ and $k, l \in \mathbb{Z}_{+}^{d}$, then $\lim _{n \rightarrow \infty} \frac{1}{n} N_{l}^{\omega}(x, n)=$ $r_{l}$ for all $l \in \mathbb{Z}_{+}^{d}$. Thus $V_{A}^{\omega} \subset U_{r}^{\omega}$ for any $A \in \mathcal{A}_{q r}$, and so

$$
H D\left(U_{r}^{\omega}\right) \geq \sup _{A \in \mathcal{A}_{q r}} H D\left(V_{A}^{\omega}\right)=H .
$$

P. Billingsley indicated to me that in order to obtain the inequality in the other direction one can employ a modification of the argument of Theorem 6 from [Bi3] based on a stronger version of Proposition 2.3 above which does not require some of the computations below. Still, this argument requires some additional work in order to adapt it to my circumstances, so I shall proceed by directly estimating the number of $(\omega, n)$-cylinders needed to cover the set $U_{r}^{\omega}$. Put $r_{l}^{\omega}(x, n)=\frac{1}{n} N_{l}^{\omega}(x, n)$, 
$q_{k}^{\omega}(n)=\frac{1}{n} M_{k}^{\omega}(n)$ (where $M_{k}^{\omega}(n)$ is the same as in $\left.(2.10)\right), d_{k l}^{\omega}(x, n)=\frac{1}{n} N_{k l}^{\omega}(x, n)$, and let $r^{\omega}(x, n)=\left(r_{l}^{\omega}(x, n)\right), q^{\omega}(n)=\left(q_{k}^{\omega}(n)\right), D^{\omega}(x, n)=\left(d_{k l}^{\omega}(x, n)\right)$ be the corresponding vectors and matrix. Since $\sum_{l \leq k} N_{k l}^{\omega}(x, n)=M_{k}^{\omega}(n)$, taking into account (1.4) I can choose an infinite probability matrix $A^{\omega}(x, n)=\left(a_{k l}^{\omega}(x, n)\right)$ so that $a_{k l}^{\omega}(x, n)=0$ unless $l \leq k$, and

$$
d_{k l}^{\omega}(x, n)=q_{k}^{\omega}(n) a_{k l}^{\omega}(x, n) \quad \text { and } \quad q^{\omega}(n) A^{\omega}(x, n)=r^{\omega}(x, n) .
$$

Let $\rho$ be a metric on the compact convex space of infinite probability matrices so that for $B=\left(b_{k l}\right)$ and $C=\left(c_{k l}\right)$,

$$
\rho(B, C)=\sum_{k, l \in \mathbb{Z}_{+}^{d}}^{\infty} 2^{-\|k\|}\left|b_{k l}-c_{k l}\right|
$$

where $\|k\|=\left|k_{1}\right|+\cdots+\left|k_{d}\right|$. Denote also

$$
\mathcal{D}_{q r}=\left\{D=\left(d_{k l}\right): d_{k l}=q_{k} a_{k l} \text { for some } A=\left(a_{k l}\right) \in \mathcal{A}_{q r}\right\} .
$$

By Birkhoff's ergodic theorem with probability one $q^{\omega}(n) \rightarrow q$ as $n \rightarrow \infty$, and by the definition $(1.5) r^{\omega}(x, n) \rightarrow r$ as $n \rightarrow \infty$ for any $x \in U_{r}^{\omega}$. This together with (2.34) yields that with probability one

$$
U_{r}^{\omega} \subset\left\{x: \lim _{n \rightarrow \infty} \rho\left(D^{\omega}(x, n), \mathcal{D}_{q r}\right)=0\right\}
$$

and, for $A=\left(a_{k l}\right)$,

$$
V_{A}^{\omega}=\left\{x: \lim _{n \rightarrow \infty} D^{\omega}(x, n)=D=\left(d_{k l}\right), d_{k l}=q_{k} a_{k l}\right\} .
$$

Since $\mathcal{A}_{q r}$ is compact, for each small $\varepsilon>0$ I can choose matrices $A^{\varepsilon, j}=\left(a_{k, l}^{\varepsilon, j}\right) \in$ $\mathcal{A}_{q r}, j=1, \ldots, J_{\varepsilon}$, such that for any $A \in \mathcal{A}_{q r}$ there exists $j$ with $\rho\left(A, A^{\varepsilon, j}\right)<\varepsilon$. Let $D^{\varepsilon, j}=\left(d_{k l}^{\varepsilon, j}\right)$; where $d_{k l}^{\varepsilon, j}=q_{k} a_{k l}^{\varepsilon, j}$ then also for any $D \in \mathcal{D}_{q r}$ there exists $j$ with $\rho\left(D, D^{\varepsilon, j}\right)<\varepsilon$. Set

$$
G_{\varepsilon, j}^{\omega}(n)=\left\{x \in \mathbb{T}^{d}: \rho\left(D^{\omega}(x, n), D^{\varepsilon, j}\right)<\varepsilon\right\} \quad \text { and } \quad G_{\varepsilon}^{\omega}(n)=\bigcup_{1 \leq j \leq J_{\varepsilon}} G_{\varepsilon, j}^{\omega}(n) .
$$

By (2.35), for $P$-a.a. $\omega$,

$$
U_{r}^{\omega} \subset \bigcup_{i=1}^{\infty} \bigcap_{n \geq i} G_{\varepsilon}^{\omega}(n)
$$

It is clear that $G_{\varepsilon, j}^{\omega}(n)$ and $G_{\varepsilon}^{\omega}(n)$ are unions of collections $\mathcal{G}_{\varepsilon, j}^{\omega}(n)$ and $\mathcal{G}_{\varepsilon}^{\omega}(n)=$ $\bigcup_{1 \leq j \leq J_{\varepsilon}} \mathcal{G}_{\varepsilon, j}^{\omega}(n)$ of $(\omega, n)$-cylinders so that

$$
\mathcal{G}_{\varepsilon, j}^{\omega}(n)=\left\{C_{\alpha_{0}, \ldots, \alpha_{n-1}}^{\omega}:\left|\frac{1}{n} \sum_{i \in I_{k}^{\omega}(n)} \chi_{l-1}\left(\alpha_{i}\right)-d_{k l}^{\epsilon, j}\right|<\varepsilon 2^{\|k\|}, \forall l \leq k ; k, l \in \mathbb{Z}_{+}^{d}\right\}
$$


where, recall, $I_{k}^{\omega}(n)$ is the set of $i \in\{1, \ldots, n\}$ such that $\left(m \circ \theta^{i}\right)(\omega)=k$. Let $\tilde{\Omega}$ be the set of all $\omega$ such that $\lim _{n \rightarrow \infty} q^{\omega}(n)=q$ and (2.19),(2.35),(2.36) hold true. Then $P(\tilde{\Omega})=1$. Fix an $\omega \in \tilde{\Omega}$. Observe that if $q_{k}=0$ then $\lim _{n \rightarrow \infty} q_{k}^{\omega}(n)=0$, and so $\lim _{n \rightarrow \infty} d_{k l}^{\omega}(x, n)=d_{k l}=0$ for any $x \in \mathbb{T}^{d}, l \leq k$, and $D=\left(d_{k l}\right) \in \mathcal{D}_{q r}$. Similarly to [Eg] and Lemma 5.3 of [Bi1,I] (see also related results in [Ca]), in order to obtain an upper bound for $H D\left(U_{r}^{\omega}\right)$ I need a good upper bound for the number of cylinders in $\mathcal{G}_{\varepsilon, j}^{\omega}(n)$. Since the number of elements in $I_{k}^{\omega}(n)$ for big $n$ is about $n q_{k}$, I can estimate the number $\sigma_{\varepsilon, j}^{\omega}(n, k)$ of choices of indices $i \in I_{k}^{\omega}(n)$ with $\alpha_{i}=l-\mathbf{1}, l \leq k$, such that the condition in braces in (2.38) holds true for a fixed $k$ (i.e. $\left|\left\{i \in I_{k}^{\omega}(n): \alpha_{i}=l-\mathbf{1}\right\}\right| \approx n d_{k l}^{\varepsilon, j}, \forall l \leq k$ ) via multinomial coefficients (cf. $[\mathrm{Eg}],[\mathrm{Bi} 1, \mathrm{I}])$,

$$
\sigma_{\varepsilon, j}^{\omega}(n, k) \leq \frac{\left[n q_{k}\right] !}{\prod_{l \leq k}\left(\left[n d_{k l}^{\varepsilon, j}\right] !\right)} e^{L_{k}^{\omega} \varepsilon n}
$$

where $0 !=1,[\cdot]$ denotes the integer part, and $L_{k}^{\omega}>0$ depends only on $k$ and $\omega$. Next, pick a big $K \in \mathbb{Z}_{+}$. I use (2.39) only for $k \leq \mathbf{K}=(K, \ldots, K) \in \mathbb{Z}_{+}^{d}$, and for all other $k$ I estimate $\sigma_{\varepsilon, j}^{\omega}(n, k)$ by the total number of choices of $\alpha_{i} \leq k$ for all $i \in I_{k}^{\omega}(n)$, which is $\prod_{a=1}^{d} k_{a}^{M_{k}^{\omega}(n)}$. Thus by (2.34) and (2.39) together with Stirling's formula I derive that for big $n$,

$$
\begin{aligned}
\frac{1}{n} \log \left|\mathcal{G}_{\varepsilon, j}^{\omega}(n)\right| \leq \frac{1}{n} \sum_{k \in \mathbb{Z}^{d}} \log \sigma_{\varepsilon, j}^{\omega}(n, k) \\
\leq \frac{1}{n} \sum_{k \leq \mathbf{K}} \log \left(\frac{\left(n q_{k} e^{-1}\right)^{n q_{k}}}{\prod_{l \leq k}\left(n d_{k l}^{\varepsilon, j} e^{-1}\right)^{n d_{k, l}^{\varepsilon, j}}} e^{2 L_{k} \varepsilon n}\right)+\frac{1}{n} \sum_{k \not \mathbf{K}} \log \prod_{i=1}^{d} k_{i}^{M_{k}^{\omega}(n)} \\
\leq \sum_{k \leq \mathbf{K}}\left(q_{k} \log q_{k}-\sum_{l \leq k} d_{k l}^{\varepsilon, j} \log d_{k l}^{\varepsilon, j}+2 L_{k}^{\omega} \varepsilon\right)+\sum_{k \not \mathbf{K}} q_{k}^{\omega}(n) \sum_{i=1}^{d} \log k_{i} \\
=-\sum_{k \leq \mathbf{K}} \sum_{l \leq k} q_{k} a_{k l}^{\varepsilon, j} \log a_{k l}^{\varepsilon, j}+2 \varepsilon \sum_{k \leq \mathbf{K}} L_{k}^{\omega}+\sum_{k \nless \mathbf{K}} q_{k}^{\omega}(n) \sum_{i=1}^{d} \log k_{i} \\
\leq H \int \log m_{1} d P+2 \varepsilon \sum_{k \leq \mathbf{K}} L_{k}^{\omega}+\sum_{k \not \mathbf{K}} q_{k}^{\omega}(n) \sum_{i=1}^{d} \log k_{i},
\end{aligned}
$$

where $H$ was defined in (2.8). Observe that

$$
\begin{gathered}
\lim _{n \rightarrow \infty} \sum_{k \nless \mathbf{K}} q_{k}^{\omega}(n) \sum_{i=1}^{d} \log k_{i}=\lim _{n \rightarrow \infty} \sum_{k \in \mathbb{Z}_{+}^{d}} q_{k}^{\omega}(n) \sum_{i=1}^{d} \log k_{i}-\lim _{n \rightarrow \infty} \sum_{k \leq \mathbf{K}} q_{k}^{\omega}(n) \sum_{i=1}^{d} \log k_{i} \\
=d \int \log m_{1} d P-\sum_{i=1}^{d} \int_{\{m \leq \mathbf{K}\}} \log m_{i} d P=\sum_{i=1}^{d} \int_{\{m \nless \mathbf{K}\}} \log m_{i} d P .
\end{gathered}
$$


For an arbitrarily small $\delta$ pick $K$ so that the right hand side of (2.41) does not exceed $\delta / 2$ and then choose first $\varepsilon$ and then $n_{0}=n_{0}^{\omega, \varepsilon}$ so that for all $n \geq n_{0}$,

$$
2 \varepsilon \sum_{k \leq \mathbf{K}} L_{k}^{\varepsilon}+\sum_{k \nless \mathbf{K}} q_{k}^{\omega}(n) \sum_{i=1}^{d} \log k_{i}+\frac{1}{n} \log J_{\varepsilon} \leq \delta .
$$

This together with (2.40) yields

$$
\frac{1}{n} \log \left|\mathcal{G}_{\varepsilon}^{\omega}(n)\right| \leq \frac{1}{n} \log \left(J_{\varepsilon} \max _{1 \leq j \leq J_{\varepsilon}}\left|\mathcal{G}_{\varepsilon, j}^{\omega}(n)\right|\right) \leq H \int \log m_{1} d P+\delta .
$$

Taking into account (2.13) and (2.19), I find that for any $\kappa>H+\delta\left(\int \log m d P\right)^{-1}$,

$$
\sum_{C \in \mathcal{G}_{\varepsilon}^{\omega}(n)}|C|^{\kappa} \leq \exp \left(n\left(H \int \log m_{1} d P+\delta-\frac{\kappa}{n} \sum_{i=1}^{n-1} \log m_{1}\left(\theta^{i} \omega\right)\right)\right)
$$

is bounded for all $n$ big enough. Since $\delta$ above is arbitrary, this together with (2.37) yields $H D\left(U_{r}^{\omega}\right) \leq H$, completing the proof of (2.8). It remains to show that $\mathcal{A}_{q r}$ is nonempty if and only if the condition (2.9) holds true. This is a corollary of the following more general result. I am indebted to B. Weiss for useful discussions and references related to it.

2.4. Proposition. Let $\mathcal{P}$ be a countable partially ordered set and $q=\left(q_{k}, k \in\right.$ $\mathcal{P}), r=\left(r_{k}, k \in \mathcal{P}\right)$ be infinite probability vectors indexed by $\mathcal{P}$. Let $\mathcal{F}$ be the collection of all filters in $\mathcal{P}$, i.e. the subsets $F$ of $\mathcal{P}$ such that if $l \in F$ and $k \geq l$ then $k \in F$. Denote by $\mathcal{A}_{q r}$ the set of all infinite probability matrices $A=\left(a_{k l} ; k, l \in \mathcal{P}\right)$ indexed by $\mathcal{P}$ such that $a_{k l}=0$ unless $k \geq l$ and $\sum_{k \in \mathcal{P}} q_{k} a_{k l}=r_{l}$ for all $l \in \mathcal{P}$. Then $\mathcal{A}_{q r}$ is not empty if and only if

$$
\sum_{k \in F} q_{k} \geq \sum_{k \in F} r_{k}, \quad \forall F \in \mathcal{F}
$$

Proof. Let $X$ and $Y$ be random variables with values in $\mathcal{P}$ such that $P\{X=k\}=q_{k}$ and $P\{Y=k\}=r_{k}$ for all $k \in \mathcal{P}$. It is easy to see that $\mathcal{A}_{q r} \neq \emptyset$ if and only if the random variables $X$ and $Y$ can be considered on one probability space (i.e. they can be coupled) so that

$$
P\{X \geq Y\}=1
$$

Indeed, if $A=\left(a_{k l} ; k, l \in \mathcal{P}\right) \in \mathcal{A}_{q r}$, then we can define the joint distribution of $X$ and $Y$ by $P\{X=k, Y=l\}=q_{k} a_{k l}$, and (2.43) will be satisfied. On the other hand, suppose we found a joint distribution of $X$ and $Y$ satisfying (2.43); then set $a_{k l}=q_{k}^{-1} P\{X=k, Y=l\}$ if $q_{k} \neq 0$ and, say, $a_{k l}=1$ for $l=k$ and $=0$ otherwise, if $q_{k}=0$. After this reduction Proposition 2.4 follows from Theorem B.1 in Chapter 17 of [MO], which, in turn, is a consequence of Theorem 11 from [St]. In case $\mathcal{P}=\mathbb{Z}_{+}^{d}$ the problem can be easily reduced to the case when $\mathcal{P}$ is finite, and then the result follows from one of corollaries of the Max-Flow Min-Cut theorem 
known as the Supply-Demand theorem (see [LP], Corollary 2.1.5). This completes the proof of Proposition 2.4, as well as Theorem 2.1 itself.

2.5. Remark. The student Z. Hellman (who participated in my course) suggested for the deterministic case the following small parameter argument, which yields also in our circumstances the required upper bound for $H D\left(V_{A}^{\omega}\right)$ when some of the $a_{k l}$ 's are zero. Let $n_{k}$ be the number of $l \leq k$ for which $a_{k l}=0$, and $\delta=\left(\delta_{k}, k \in \mathbb{Z}_{+}^{d}\right)$ be an infinite vector with positive entries $\delta_{k}$ which are so small that if $n_{k} \geq 1$ then $a_{k l}-\delta_{k} n_{k}^{-1}>0$ provided $a_{k l}>0$, and

$$
-\log \left(\delta_{k} n_{k}^{-1}\right) \geq-n_{k}^{-1} \sum_{l \leq k, a_{k l}>0} \log \left(a_{k l}-\delta_{k} n_{k}^{-1}\right) .
$$

If $n_{k}=0$ set $a_{k l}^{(\delta)}=a_{k l}$, otherwise put $a_{k l}^{(\delta)}=a_{k l}-\delta_{k} n_{k}^{-1}$ if $a_{k l}>0$ and $a_{k l}^{(\delta)}=\delta_{k} n_{k}^{-1}$ if $a_{k l}=0$. Then

$$
\sum_{l \leq k, a_{k l}>0} a_{k l} \log a_{k l}^{(\delta)} \geq \sum_{l \leq k} a_{k l}^{(\delta)} \log a_{k l}^{(\delta)},
$$

and so if $A^{(\delta)}=\left(a_{k l}^{(\delta)}\right)$ and

$$
W_{A^{(\delta)}}^{\omega}=\left\{x: \limsup _{n \rightarrow \infty}\left(-\frac{1}{n} \log \nu_{p_{A^{(\delta)}}}^{\omega}\left(C_{n}^{\omega}(x)\right)\right) \leq-H_{A^{(\delta)}} \int \log m_{1} d P\right\}
$$

then with probability one,

$$
V_{A}^{\omega} \subset\left\{x: \lim _{n \rightarrow \infty}\left(-\frac{1}{n} \log \nu_{p_{A^{(\delta)}}}^{\omega}\left(C_{n}^{\omega}(x)\right)\right)=-\sum_{k \in \mathbb{Z}_{+}^{d}} q_{k} \sum_{l \leq k} a_{k l} \log a_{k l}^{(\delta)}\right\} \subset W_{A^{(\delta)}}^{\omega} .
$$

From here, (2.24), and Birkhoff's ergodic theorem it follows as above that with probability one $H D\left(V_{A}^{\omega}\right) \leq H D\left(W_{A^{(\delta)}}^{\omega}\right) \leq H_{A^{(\delta)}}$. Letting $\delta \rightarrow 0$ (i.e. $\delta_{k} \rightarrow 0$ for all $\left.k \in \mathbb{Z}_{+}^{d}\right)$, one obtains the required upper bound $H D\left(V_{A}^{\omega}\right) \leq H_{A}$.

2.6. Remark. The supremum in (2.8) of the expression in (2.7) over $\mathcal{A}_{q r}$ can be found, in principle, employing the Lagrange multipliers method, though it does not seem easy to find a nice general analytical formula for $H$ since we also have constraints on $A=\left(a_{k l}\right) \in \mathcal{A}_{q r}$ given by inequalities $a_{k l} \geq 0$, and so the maximum may be attained on the boundary of $\mathcal{A}_{q r}$.

2.7. Remark. The limit in braces in (2.31) gives the relativised entropy of $\tau$ with respect to the measure $\nu_{p_{A}}$, and this is connected with the relativized ShannonMcMillan-Breiman theorem (see $[\mathrm{Bo}]$ ).

2.8. Remark. In the same way as in $[\mathrm{Sm}]$, using general iterated logarithm type theorems from $[\mathrm{Ph}]$ one can show that with probability one the Hausdorff measure in dimension $H_{A}$ of the sets $V_{A}^{\omega}$ equals infinity except for the case when $H_{A}=d$.

2.9. Remark. Though $[\mathrm{KK}]$ contains a general proof of ergodicity of Gibbs measures for the skew product transformation $\tau$, I give here for the reader's convenience a 
direct independent proof of this fact. Observe that if $j_{1}, j_{2}, n \in \mathbb{Z}_{+}$and $n \geq j_{1}$, then

$$
\begin{aligned}
& \nu_{p}^{\omega}\left(C_{\alpha_{0}, \ldots, \alpha_{j_{1}-1}}^{\omega} \bigcap\left(f^{n}(\omega)\right)^{-1} C_{\beta_{0}, \ldots, \beta_{j_{2}-1}}^{\theta^{n} \omega}\right) \\
& \quad=\prod_{i=0}^{j_{1}-1} p_{\alpha_{i}}^{\theta^{i} \omega}\left(\sum_{\gamma_{0}, \ldots, \gamma_{n-j_{1}}} \prod_{i=0}^{n-j_{1}} p_{\gamma_{i}}^{\theta^{k+i} \omega}\right) \prod_{i=0}^{j_{2}-1} p_{\beta_{i}}^{\theta^{n+i} \omega} \\
& \quad=\nu_{p}^{\omega}\left(C_{\alpha_{0}, \ldots, \alpha_{j_{1}-1}}^{\omega}\right) \nu_{p}^{\theta^{n} \omega}\left(C_{\beta_{0}, \ldots, \beta_{j_{2}-1}}^{\theta^{n} \omega}\right) .
\end{aligned}
$$

Approximating sets by disjoint unions of cylinder sets, I derive that for any measurable collection of measurable sets $\Gamma^{\omega} \subset \mathbb{T}^{d}$,

$$
\nu_{p}^{\omega}\left(\Gamma^{\omega} \cap\left(f^{n}(\omega)\right)^{-1} \Gamma^{\theta^{n} \omega}\right)-\nu_{p}^{\omega}\left(\Gamma^{\omega}\right) \nu_{p}^{\theta^{n} \omega}\left(\Gamma^{\theta^{n} \omega}\right) \longrightarrow 0 \quad \text { as } n \rightarrow \infty .
$$

Now suppose that $\Gamma \subset \mathbb{T}^{d} \times \Omega$ is a measurable set and $\tau^{-1} \Gamma=\Gamma$. Then for $\Gamma^{\omega}=\{x:(x, \omega) \in \Gamma\}$ it follows that

$$
\left(f^{n}(\omega)\right)^{-1} \Gamma^{\theta^{n} \omega}=\Gamma^{\omega} .
$$

Since $\nu_{p}$ is $\tau$-invariant, then, by (2.30) and (2.44) for $P$-a.a. $\omega$,

$$
\nu_{p}^{\theta^{n} \omega}\left(\Gamma^{\theta^{n} \omega}\right)=\left(f^{n}(\omega) \nu_{p}^{\omega}\right)\left(\Gamma^{\theta^{n} \omega}\right)=\nu_{p}^{\omega}\left(\left(f^{n}(\omega)\right)^{-1} \Gamma^{\theta^{n} \omega}\right)=\nu_{p}^{\omega}\left(\Gamma^{\omega}\right),
$$

and so by (2.43) and (2.44), for $P$-a.a. $\omega$,

$$
\nu_{p}^{\omega}\left(\Gamma^{\omega}\right)=\left(\nu_{p}^{\omega}\left(\Gamma^{\omega}\right)\right)^{2} .
$$

Hence, $\nu_{p}^{\omega}\left(\Gamma^{\omega}\right)=0$ or 1 for $P$-a.a. $\omega$, which together with (2.45) and ergodicity of $P$ with respect to $\theta$ yield, that either $\nu_{p}^{\omega}\left(\Gamma^{\omega}\right)=0$ for $P$-a.a. $\omega$ or $\nu_{p}^{\omega}\left(\Gamma^{\omega}\right)=1$ for $P$-a.a. $\omega$, and so $\nu_{p}(\Gamma)=0$ or 1 , completing the proof of ergodicity of $\nu_{p}$.

2.10. Example. Let $d=1, q_{2}=q_{3}=1 / 2$, and $r_{1}=r_{2}=r_{3}=1 / 3$ with all other $q_{i}$ and $r_{i}$ equal zero. Then $\mathcal{A}_{q r}$ consists of all matrices $A=\left(a_{k l}\right)$ such that $a_{33}=2 / 3, a_{21}=\gamma, a_{22}=1-\gamma, a_{31}=2 / 3-\gamma, a_{32}=\gamma-1 / 3, a_{i i}=a_{11}=1, \forall i>3$, where $1 / 3 \leq \gamma \leq 2 / 3$ and all other $a_{i j}$ equal zero. Then

$$
\begin{array}{r}
H_{A}=-\frac{1}{2}(\gamma \log \gamma+(1-\gamma) \log (1-\gamma)+(2 / 3-\gamma) \log (2 / 3-\gamma) \\
+(\gamma-1 / 3) \log (\gamma-1 / 3)+2 / 3 \log 2 / 3) .
\end{array}
$$

Maximizing $H_{A}$ in $\gamma \in[1 / 3,2 / 3]$, I obtain that

$$
H=H D\left(U_{r}^{\omega}\right)=1-\frac{\log 2}{3(\log 2+\log 3)},
$$

which is the absolute maximum here since it is attained at $\gamma=1 / 2$ inside of our domain.

2.11. Example. Let $d=1, q_{2}=1 / 3, q_{3}=2 / 3$, and $r_{1}=r_{3}=1 / 2$ with all other $q_{i}$ and $r_{i}$ equal to zero, which corresponds to a "random Cantor set". Then $\mathcal{A}_{q r}$ consists of only one matrix $A=\left(a_{k l}\right)$ such that $a_{31}=1 / 4, a_{33}=3 / 4, a_{11}=a_{21}=$ $a_{i i}=1, \forall i>3$ and all other $a_{i j}$ equal zero. Then

$$
H=H_{A}=\frac{2 \log 2-\frac{3}{4} \log 3}{\log 2+2 \log 3} .
$$




\section{Generalizations And Related Results}

Next, similarly to [Bi1] I shall study frequencies of pairs of consecutive "vectordigits" in the expansion (1.2). For any $\bar{k}=\left(k^{(1)}, k^{(2)}\right), \bar{l}=\left(l^{(1)}, l^{(2)}\right) \in \mathbb{Z}_{+}^{2 d}$, where $k^{(i)}, l^{(i)} \in \mathbb{Z}_{+}^{d}, i=1,2$, set

$$
\begin{array}{r}
N_{\bar{k} \bar{l}}^{\omega}(x, n)=\mid\left\{i \geq 0, i<n: m\left(\theta^{i} \omega\right)=k^{(1)}, m\left(\theta^{i+1} \omega\right)=k^{(2)},\right. \\
\left.x_{i}(\omega)=l^{(1)}-\mathbf{1}, x_{i+1}(\omega)=l^{(2)}-\mathbf{1}\right\} \mid
\end{array}
$$

and

$$
N_{\bar{l}}^{\omega}(x, n)=\sum_{\bar{k} \in \mathbb{Z}_{+}^{2 d}} N_{\bar{k} l}^{\omega} .
$$

Let $r=\left(r_{\bar{k}}, \bar{k} \in \mathbb{Z}_{+}^{2 d}\right)$ be an infinite probability vector and $A=\left(a_{\bar{k} \bar{l}}, \bar{k}, \bar{l} \in \mathbb{Z}_{+}^{2 d}\right)$ be an infinite probability matrix such that $a_{\bar{k} \bar{l}}=0$ unless $\bar{l} \leq \bar{k}$ (i.e. $l^{(i)} \leq k^{(i)}, i=$ $1,2)$. Set

$$
U_{r}^{\omega}=\left\{x \in \mathbb{T}^{d}: \lim _{n \rightarrow \infty} \frac{1}{n} N_{\bar{l}}^{\omega}(x, n)=r_{\bar{l}} \text { for all } \bar{l} \in \mathbb{Z}_{+}^{2 d}\right\}
$$

and

$$
V_{A}^{\omega}=\left\{x \in \mathbb{Z}_{+}^{d}: \lim _{n \rightarrow \infty} \frac{1}{n} N_{\bar{k} \bar{l}}^{\omega}(x, n)=q_{\bar{k}} a_{\bar{k} \bar{l}} \text { for all } \bar{k}, \bar{l} \in \mathbb{Z}_{+}^{2 d}\right\},
$$

where $q_{\bar{k}}=P\left\{m=k^{(1)}, m \circ \theta=k^{(2)}\right\}, \bar{k}=\left(k^{(1)}, k^{(2)}\right)$.

3.1. Theorem. With probability one,

$$
H D\left(V_{A}^{\omega}\right)=\frac{-\sum_{\bar{k} \in \mathbb{Z}_{+}^{22}} q_{\bar{k}} \sum_{\bar{l} \leq \bar{k}} a_{\bar{k} \bar{l}} \log \left(a_{\bar{k} \bar{l}} a_{\bar{k} l^{(1)}}^{-1}\right)}{\int \log m_{1} d P} \stackrel{\text { def }}{=} H_{A},
$$

where $a_{\bar{k} l^{(1)}}=\sum_{l^{(2)} \in \mathbb{Z}_{+}^{d}} a_{k l}$ if $l=\left(l^{(1)}, l^{(2)}\right)$ with the convention $0 \log 0=0$. Furthermore, for P-a.a. $\omega$,

$$
H D\left(U_{r}^{\omega}\right)=\sup _{A \in \mathcal{A}_{q r}} H_{A} \stackrel{\text { def }}{=} H
$$

where the supremum in (3.6) is taken over the set $\mathcal{A}_{q r}$ of all infinite probability matrices $A=\left(a_{\bar{k} \bar{l}}\right)$ such that $a_{\bar{k} \bar{l}}=0$ unless $\bar{l} \leq \bar{k}$ and $q A=r$. The set $\mathcal{A}_{q r}$ is nonempty if and only if

$$
\sum_{\bar{l} \in F} q_{\bar{l}} \geq \sum_{\bar{l} \in F} r_{\bar{l}}
$$

for any filter $F$ in $\mathbb{Z}_{+}^{2 d}$. If (3.7) does not hold true for some filter $F$, then with probability one $U_{r}^{\omega}$ is empty.

Proof. The argument remains the same as in Theorem 2.1 except that now I have to use random Markov measures in place of random Bernoulli measures. Namely, set 
$p_{\bar{l}}^{\omega}=p_{l^{(1)}, l^{(2)}}^{\omega}=a_{\bar{k} \bar{l}} a_{\bar{k} l^{(1)}}^{-1}, \bar{k}=\left(k^{(1)}, k^{(2)}\right)$ and $p_{l^{(1)}}^{\omega}=a_{\bar{k} l^{(1)}}$ if $m(\omega)=k^{(1)}, m(\theta \omega)=$ $k^{(2)}$, where $a_{\bar{k} l^{(1)}}$ is the same as above. By the Kolmogorov extension theorem there exist a family of measures $\nu_{p_{A}}^{\omega}, \omega \in \Omega$, such that for any $(n, \omega)$-cylinder,

$$
\nu_{p_{A}}^{\omega}\left(C_{\alpha_{0}, \ldots, \alpha_{n-1}}^{\omega}\right)=p_{\alpha_{0}+\mathbf{1}}^{\omega} p_{\alpha_{0}+\mathbf{1}, \alpha_{1}+\mathbf{1}}^{\omega} \cdots p_{\alpha_{n-2}+\mathbf{1}, \alpha_{n-1}+\mathbf{1}}^{\theta^{n-2} \omega}
$$

and so

$$
\begin{gathered}
-\frac{1}{n} \log \nu_{p_{A}}^{\omega}\left(C_{n}^{\omega}(x)\right)=-\frac{1}{n} \log p_{x_{0}(\omega)+\mathbf{1}}^{\omega}-\frac{1}{n} \sum_{i=0}^{n-2} \log p_{x_{i}(\omega)+\mathbf{1}, x_{i+1}(\omega)+\mathbf{1}}^{\theta^{i} \omega} \\
=-\frac{1}{n} \log p_{x_{0}(\omega)+\mathbf{1}}^{\omega}-\frac{1}{n} \sum_{\bar{k} \in \mathbb{Z}^{2 d}} \sum_{\bar{l} \leq \bar{k}} N_{\bar{k} \bar{l}}^{\omega}(x, n) \log \left(a_{\bar{k} \bar{l}} a_{\bar{k} l^{(1)}}^{-1}\right) .
\end{gathered}
$$

The measure $\nu_{p_{A}}$ defined on $\mathbb{T}^{d} \times \Omega$ by $\nu_{p_{A}}(W)=\int \nu_{p_{A}}^{\omega}\left(W^{\omega}\right) d P(\omega), W^{\omega}=\{x \in$ $\left.\mathbb{T}^{d}:(x, \omega) \in W\right\}$, is the unique equilibrium state (Gibbs measure) corresponding to the function $\psi(x, \omega)=\log p_{x_{0}(\omega), x_{1}(\omega)}^{\omega}$ (called, naturally, a random Markov measure), and so it is ergodic (see $[\mathrm{KK}]$ ). Thus I can proceed verbatim in the same way as in Theorem 2.1 in order to complete the proof of Theorem 3.1.

Next, let $R$ be a compact set of infinite probability vectors $r=\left(r_{\bar{k}}, \bar{k} \in \mathbb{Z}_{+}^{2 d}\right)$ and $\mathcal{A}$ be a compact set of infinite probability matrices $A=\left(a_{\bar{k} \bar{l}}, \bar{k}, \bar{l} \in \mathbb{Z}_{+}^{2 d}\right)$ such that $a_{\bar{k} \bar{l}}=0$ unless $\bar{l} \leq \bar{k}$. In the same way as in Section 2 set $r_{\bar{l}}^{\omega}(x, n)=$ $\frac{1}{n} N_{\bar{l}}^{\omega}(x, n), d_{\bar{k} \bar{l}}^{\omega}(x, n)=\frac{1}{n} N_{\bar{k} \bar{l}}^{\omega}(x, n)$ and let $r^{\omega}=\left(r_{\bar{l}}^{\omega}\right), D^{\omega}(x, n)=\left(d_{\bar{k} \bar{l}}^{\omega}\right)$ be the corresponding vector and matrix. Denote also $\mathcal{D}=\left\{D=\left(d_{\bar{k} \bar{l}}\right): d_{\bar{k} \bar{l}}=q_{\bar{k}} a_{\bar{k} \bar{l}}\right.$ and $A=$ $\left.\left(a_{\bar{k} \bar{l}}\right) \in \mathcal{A}\right\}$. Denote by $\rho$ the distances between corresponding vectors and matrices, so that $\rho(r, \tilde{r})=\sum_{\bar{k} \in \mathbb{Z}_{+}^{2 d}}\left|r_{\bar{k}}-\tilde{r}_{\bar{k}}\right|$ and $\rho(B, C)=\sum_{\bar{k}, \bar{l} \in \mathbb{Z}_{+}^{2 d}} 2^{-\|\bar{k}\|}\left|b_{\bar{k} \bar{l}}-c_{\bar{k} \bar{l}}\right|$, where $\|k\|=\left\|k^{(1)}\right\|+\left\|k^{(2)}\right\|$ if $k=\left(k^{(1)}, k^{(2)}\right)$. Next, define the sets

$$
U_{R}^{\omega}=\left\{x \in \mathbb{T}^{d}: \lim _{n \rightarrow \infty} \rho\left(r^{\omega}(x, n), R\right)=0\right\}
$$

and

$$
V_{\mathcal{A}}^{\omega}=\left\{x \in \mathbb{T}^{d}: \lim _{n \rightarrow \infty} \rho\left(D^{\omega}(x, n), \mathcal{D}\right)=0\right\} .
$$

In the same way as I derived (2.8) from (2.7) I obtain

3.2. Theorem. With probability one,

$$
H D\left(U_{R}^{\omega}\right)=\sup _{\bar{r} \in R} H D\left(U_{\bar{r}}^{\omega}\right) \quad \text { and } \quad H D\left(V_{\mathcal{A}}^{\omega}\right)=\sup _{A \in \mathcal{A}} H D\left(V_{A}^{\omega}\right)
$$

where the right hand side of (3.11) is given by Theorem 3.1.

Next, I shall consider another version of Theorem 2.1 where the condition (1.1) will be relaxed but $U_{r}^{\omega}$ and $V_{A}^{\omega}$ will be product sets generalizing Example 6 in [Bi3]. Namely, let

$$
0<\gamma_{i}=\int \log m_{i} d P<\infty, \quad i=1, \ldots, d
$$


but the $\gamma_{i}$ 's may be different now. For $k, l \in \mathbb{Z}_{+}$and $i=1, \ldots, d$ set

$$
\begin{aligned}
& N_{k l}^{\omega, i}(x, n)=\left|\left\{j \geq 0, j<n: m_{i}\left(\theta^{j} \omega\right)=k, x_{i j}(\omega)=l-1\right\}\right|, \\
& N_{l}^{\omega, i}(x, n)=\sum_{k=1}^{\infty} N_{k l}^{\omega, i}(x, n),
\end{aligned}
$$

where $m(\omega)$ and $x_{j}(\omega)$ are the same as in the Introduction. Let $r^{(i)}=\left(r_{k}^{(i)} ; k \in \mathbb{Z}_{+}\right)$ and $A^{(i)}=\left(a_{k l}^{(i)} ; k, l \in \mathbb{Z}_{+}\right), i=1, \ldots, d$, be infinite probability vectors and matrices, respectively. Denote by $\pi_{i}$ the projection to the $i$-th coordinate on $\mathbb{T}^{d}$. Set

$$
U_{r^{(i)}}^{\omega}=\left\{\pi_{i} x: x \in \mathbb{T}^{d}, \lim _{n \rightarrow \infty} \frac{1}{n} N_{l}^{\omega, i}(x, n)=r_{l}^{(i)}, \forall l \in \mathbb{Z}_{+}\right\}
$$

and

$$
V_{A^{(i)}}^{\omega}=\left\{\pi_{i} x: x \in \mathbb{T}^{d}, \lim _{n \rightarrow \infty} N_{k l}^{\omega, i}(x, n)=q_{k}^{(i)} a_{k l}^{(i)} \forall k, l \in \mathbb{Z}_{+}\right\}
$$

where $q_{k}^{(i)}=P\left\{m_{i}=k\right\}$. For $\alpha_{0}, \ldots, \alpha_{n-1} \in \mathbb{Z}_{+}$I consider also the one dimensional $(\omega, n)$-cylinders

$$
C_{\alpha_{0}, \ldots, \alpha_{n-1}}^{\omega, i}=\left\{\pi_{i} x: x_{i j}(\omega)=\alpha_{j} \forall j=0, \ldots, n-1\right\} .
$$

Let $n=\left(n_{1}, \ldots, n_{d}\right)$, where $n_{i}=\left[n_{1} \frac{\gamma_{1}}{\gamma_{i}}\right]$. Then in the same way as in Lemma 2.2 it follows that the Hausdorff dimension of any subset of $\mathbb{T}^{d}$ can be computed considering only covers by the product sets

$$
C_{\alpha, n}^{\omega}=\prod_{i=1}^{d} C_{\alpha_{0}^{(i)}, \ldots, \alpha_{n_{i}-1}^{(i)}}^{\omega, i} .
$$

Let $p_{A}^{\omega, i}=\left(p_{l}^{\omega, i} ; l \in \mathbb{Z}_{+}\right), i=1, \ldots, d$, be infinite probability vectors such that $\sum_{l=1}^{m_{i}(\omega)} p_{l}^{\omega, i}=1$ and $p_{l}^{\omega, i}=a_{k l}^{(i)}$ if $\omega \in\left\{\omega: m_{i}(\omega)=k\right\}$. Then by the Kolmogorov theorem there exist probability measures $\nu_{p_{A}}^{\omega, i}$ on $[0,1)$ such that

$$
\nu_{p_{A}}^{\omega, i}\left(C_{\alpha_{0}, \ldots, \alpha_{n-1}}^{\omega,,}\right)=\prod_{j=0}^{n-1} p_{\alpha_{j}+1}^{\theta^{j} \omega, i}
$$

Let $\nu_{p_{A}}^{\omega}=\prod_{i=1}^{d} \nu_{p_{A}}^{\omega, i}$ be the product measure. Denote by $C_{n}^{\omega}$ the set $C_{\alpha, n}^{\omega}$ containing a point $x$. Then

$$
\begin{aligned}
-\frac{1}{n_{1}} \log \nu_{p_{A}}^{\omega}\left(C_{n}^{\omega}(x)\right) & =-\frac{1}{n_{1}} \sum_{i=1}^{d} \sum_{j=0}^{n_{i}-1} \log p_{x_{i j}(\omega)+1}^{\theta^{j} \omega,} \\
& =-\frac{1}{n_{1}} \sum_{i=1}^{d} \sum_{k=1}^{\infty} \sum_{l=1}^{k} N_{k l}^{\omega, i}\left(x, n_{i}\right) \log a_{k l}^{(i)} .
\end{aligned}
$$


By the ergodic theorem, with probability one,

$$
\lim _{n_{1} \rightarrow \infty} \frac{1}{n_{1}} \log \operatorname{Leb}\left(C_{n}^{\omega}(x)\right)=\lim _{n_{1} \rightarrow \infty} \sum_{i=1}^{d} \sum_{j=0}^{n_{i}-1} \log \frac{1}{m_{i}\left(\theta^{j} \omega\right)}=\gamma_{1} d
$$

and for any $x \in V_{A^{(i)}}^{\omega}$,

$$
\lim _{n_{1} \rightarrow \infty} \frac{1}{n_{1}} N_{k l}^{\omega, i}\left(x, n_{i}\right)=\frac{\gamma_{1}}{\gamma_{i}} q_{k}^{(i)} a_{k l}^{(i)}
$$

Thus

$$
V_{A^{(1)}}^{\omega} \times V_{A^{(2)}}^{\omega} \times \cdots \times V_{A^{(d)}}^{\omega} \subset\left\{x: \lim _{n \rightarrow \infty} \frac{\log \nu_{p_{A}}^{\omega}\left(C_{\alpha, n}^{\omega}(x)\right)}{\log \operatorname{Leb}\left(C_{\alpha, n}^{\omega}(x)\right)}=\sum_{i=1}^{d} H_{A^{(i)}}\right\}
$$

where

$$
H_{A^{(i)}}=-\gamma_{i}^{-1} \sum_{k=1}^{\infty} q_{k}^{(i)} \sum_{l=1}^{k} a_{k l}^{(i)} \log a_{k l}^{(i)}
$$

Now similarly to Section 2 I derive from (3.17)-(3.20) the following result.

3.3. Theorem. With probability one,

$$
H D\left(V_{A^{(1)}}^{\omega} \times \cdots \times V_{A^{(d)}}^{\omega}\right)=\sum_{i=1}^{d} H_{A^{(i)}}
$$

and

$$
H D\left(U_{r^{(1)}}^{\omega} \times \cdots \times U_{r^{(d)}}^{\omega}\right)=\sup _{A^{(i)}, i=1, \ldots, d} \sum_{i=1}^{d} H_{A^{(i)}}
$$

where the supremum is taken over the probability matrices $A^{(i)}=\left(a_{k l}^{(i)} ; k, l \in \mathbb{Z}_{+}\right)$ such that $a_{k l}^{(i)}=0$ unless $l \leq k$ and $\sum_{k=1}^{\infty} q_{k}^{(i)} a_{k l}^{(i)}=r_{l}^{(i)}, \forall i=1, \ldots, d$.

Finally, I shall discuss large deviations for $\frac{1}{n} N_{\bar{k} \bar{l}}^{\omega}(x, n)$ and $\frac{1}{n} N_{\bar{l}}^{\omega}(x, n)$. Introduce a family of probability measures on $\mathbb{T}^{d} \times \Omega$ by

$$
\zeta_{x, \omega}^{n}=\sum_{i=0}^{n-1} \delta_{\tau^{i}(x, \omega)},
$$

where $\delta_{(x, \omega)}$ denotes the unit mass at $(x, \omega)$ and $\tau$ was defined by (1.8). Since Leb is a partial case of a random Bernoulli measure (see Section 2), and so it is an equilibrium state for $\tau$, then the thermodynamic formalism for the random expanding transformations $f(\omega), \omega \in \Omega$ exhibited in [Ki2],[KK], and [BG2] yields in view of Theorem $\mathrm{D}$ in [Ki2] the following relativized large deviations bounds. 
3.4. Theorem. Let $\Omega$ be a compact metric space. Then with probability one, for any closed $K \subset \mathcal{P}\left(\mathbb{T}^{d} \times \Omega\right)$,

$$
\limsup _{n \rightarrow \infty} \frac{1}{n} \log \operatorname{Leb}\left\{x \in \mathbb{T}^{d}: \zeta_{x, \omega}^{n} \in K\right\} \leq-\inf _{\nu \in K} I(\nu),
$$

and for any open $G \subset \mathcal{P}\left(\mathbb{T}^{d} \times \Omega\right)$,

$$
\liminf _{n \rightarrow \infty} \frac{1}{n} \log \operatorname{Leb}\left\{x \in \mathbb{T}^{d}: \zeta_{x, \omega}^{n} \in G\right\} \geq-\inf _{\nu \in G} I(\nu)
$$

where $\mathcal{P}(M)$ denotes the space of probability measures on $M$ with the topology of weak convergence, $I(\nu)=-d \int \log m_{1} d P-h_{\nu}^{(r)}(\tau)$ if $\nu$ is $\tau$-invariant with the marginal on $\Omega$ equal to $P$, and $I(\nu)=\infty$, otherwise. Here $h_{\nu}^{(r)}(\tau)$ denotes the relativized entropy of $\tau$ with respect to $\nu$ (see [Ki2] and [Bo]).

Applying the contraction principle arguments (see [DZ], Section 4.2) with respect to an appropriate topology, one can derive from Theorem 3.4 that with probability one, for any $-\infty \leq a<b \leq \infty$,

$$
\limsup _{n \rightarrow \infty} \frac{1}{n} \log \operatorname{Leb}\left\{x \in \mathbb{T}^{d}: \frac{1}{n} N_{\bar{l}}^{\omega}(x, n) \in[a, b]\right\} \leq-\inf _{c \in[a, b]} J_{\bar{l}}^{(1)}(c),
$$

$$
\liminf _{n \rightarrow \infty} \frac{1}{n} \log \operatorname{Leb}\left\{x \in \mathbb{T}^{d}: \frac{1}{n} N_{\bar{l}}^{\omega}(x, n) \in(a, b)\right\} \geq-\inf _{c \in(a, b)} J_{\bar{l}}^{(1)}(c),
$$

$$
\limsup _{n \rightarrow \infty} \frac{1}{n} \log \operatorname{Leb}\left\{x \in \mathbb{T}^{d}: \frac{1}{n} N_{\bar{k} \bar{l}}^{\omega}(x, n) \in[a, b]\right\} \leq-\inf _{c \in[a, b]} J_{\bar{k} \bar{l}}^{(2)}(c),
$$

and

$$
\liminf _{n \rightarrow \infty} \frac{1}{n} \log \operatorname{Leb}\left\{x \in \mathbb{T}^{d}: \frac{1}{n} N_{\bar{k} \bar{l}}^{\omega}(x, n) \in(a, b)\right\} \geq-\inf _{c \in(a, b)} J_{\bar{k} \bar{l}}^{(2)}(c)
$$

where

$$
J_{\bar{l}}^{(1)}(c)=\inf \left\{I(\nu): \int \chi_{l^{(1)}-\mathbf{1}}\left(x_{0}(\omega)\right) \chi_{l^{(2)}-\mathbf{1}}\left(x_{1}(\omega)\right) d \nu(x, \omega)=c\right\}
$$

and

$$
\begin{aligned}
J_{\bar{k} \bar{l}}^{(2)}(c)=\inf \{ & I(\nu): \int \chi_{k^{(1)}}(m(\omega)) \\
& \left.\times \chi_{k^{(2)}}(m(\theta \omega)) \chi_{l^{(1)}-\mathbf{1}}\left(x_{0}(\omega)\right) \chi_{l^{(2)}-\mathbf{1}}\left(x_{1}(\omega)\right) d \nu(x, \omega)=c\right\} .
\end{aligned}
$$

A delicate point in the application of the contraction principle here is that the functions $\varphi_{\bar{l}}^{\omega}(x)=\chi_{\bar{l}}\left(x_{0}(\omega)\right)$ are not continuous in $x \in \mathbb{T}^{d}$ when $\omega$ is fixed with respect to the usual topology of $\mathbb{T}^{d}$. One can overcome this taking into account that the points of discontinuity of $\varphi_{\bar{l}}^{\omega}$ in $\mathbb{T}^{d}$ have zero measure with respect to $P$ a.a. disintegrations of all equilibrium states of $\tau$ corresponding to a large class of functions (see $[\mathrm{KK}]$ ) and the set of such equilibrium states is dense in $\mathcal{P}\left(\mathbb{T}^{d} \times \Omega\right)$ (see [Ki1]). This is connected also with the symbolic representation of the random dynamical system $\tau=(f(\omega), \theta)$ as a random subshift of finite type (see [BG1,2] and $[\mathrm{KK}])$. 


\section{Dimensions OF RANDOM REPELLERS}

Let $K^{\omega}, \omega \in \Omega$, be a measurable family of compact subsets of $\mathbb{T}^{d}$ satisfying (1.10) with $f(\omega)$ the same as in the Introduction. Set $K=\left\{(x, \omega): x \in K^{\omega}\right\}$. Then we have the setup of "random bundle maps" from [BG2], and can speak about the relativized topological entropy $h_{\text {top }}^{(r)}(\tau, K)$ of the skew product transformation $\tau$ restricted to the $\tau$-invariant set $K$.

4.1. Theorem. With probability one,

$$
H D\left(K^{\omega}\right)=\frac{h_{\mathrm{top}}^{(r)}(\tau, K)}{\int \log m_{1} d P}
$$

Proof. The relativized variational principle from [Bo] (see also [LW], where $\Omega$ was supposed to be compact) can be easily generalized to this setup, yielding that for any $\varepsilon>0$ there exists a $\tau$-invariant ergodic probability measure $\nu_{\varepsilon}$ on $K$ such that $d \nu_{\varepsilon}(x, \omega)=d \nu_{\varepsilon}^{\omega}(x) d P(\omega)$, with probability one $\nu_{\varepsilon}^{\omega}\left(K^{\omega}\right)=1$ and $f(\omega) \nu_{\varepsilon}^{\omega}=\nu_{\varepsilon}^{\theta \omega}$, and

$$
h_{\nu_{\varepsilon}}^{(r)}(\tau) \geq h_{\mathrm{top}}^{(r)}-\varepsilon
$$

where $h_{\nu}^{(r)}(\tau)$ denotes the relativized metric entropy of $\tau$ with respect to a $\tau$ invariant measure $\nu$ (see $[\mathrm{Bo}]$ ). Then by the relativized Shannon-McMillan-Breiman theorem (see $[\mathrm{Bo}])$, for $\nu_{\varepsilon}$-a.a. $(x, \omega)$,

$$
\lim _{n \rightarrow \infty}\left(-\frac{1}{n} \log \nu_{\varepsilon}^{\omega}\left(C_{n}^{\omega}(x)\right)\right)=h_{\nu_{\varepsilon}}^{(r)}(\tau)
$$

and by the Fubini theorem, for $P$-a.a. $\omega$,

$$
\nu_{\varepsilon}^{\omega}\left\{x \in K^{\omega}: \lim _{n \rightarrow \infty}\left(-\frac{1}{n} \log \nu^{\omega}\left(C_{n}^{\omega}(x)\right)\right)=h_{\nu_{\varepsilon}}^{(r)}(\tau)\right\}=1,
$$

where $C_{n}^{\omega}(x)$ was defined before Proposition 2.3. Denote the set in braces in (4.4) by $K_{0}^{\omega}$. Then by Proposition 2.3, in the same way as in Section 2, I derive from (4.2)-(4.4),

$$
H D\left(K^{\omega}\right) \geq H D\left(K_{0}^{\omega}\right)=\frac{h_{\nu_{\varepsilon}}^{(r)}(\tau)}{\int \log m_{1} d P} \geq \frac{h_{\mathrm{top}}^{(r)}-\varepsilon}{\int \log m_{1} d P} .
$$

Since $\varepsilon$ here is arbitrary, I obtain that the left hand side of (4.1) is not less than the right hand side of (4.1). In order to obtain an upper bound for $H D\left(K^{\omega}\right)$ I shall need the following result, which is contained, essentially, in [BG2] and [KK].

4.2. Lemma. With probability one,

$$
h_{\text {top }}^{(r)}(\tau, K)=\lim _{n \rightarrow \infty} \frac{1}{n} \log r_{n}^{\omega}(K)
$$

where

$$
r_{n}^{\omega}(K)=\left|\left\{C_{\alpha_{0}, \ldots, \alpha_{n-1}}^{\omega}: C_{\alpha_{0}, \ldots, \alpha_{n-1}}^{\omega} \cap K^{\omega} \neq \emptyset\right\}\right| .
$$


Proof. For any $x, y \in \mathbb{T}^{d}$ set $\rho_{n}^{\omega}(x, y)=\max _{0 \leq j \leq n-1} \operatorname{dist}\left(f^{j}(\omega) x, f^{j}(\omega) y\right)$, where $f^{j}(\omega)=f\left(\theta^{j} \omega\right) \circ \cdots \circ f(\theta \omega) \circ f(\omega)$, and denote $B_{\delta}^{\omega}(x, n)=\left\{y \in \mathbb{T}^{d}: \rho_{n}^{\omega}(x, y) \leq \delta\right\}$. A subset $E \subset K^{\omega}$ is called $(\omega, \delta, n)$-separated if for any $x, y \in E, x \neq y$, one has $\rho_{n}^{\omega}(x, y)>\delta$. If $E_{n}^{\omega}$ is any family of maximal $(\omega, \delta, n)$-separated sets, then with probability one (see [Ki1], [Bo], [KK]),

$$
h_{\mathrm{top}}^{(r)}(\tau, K)=\lim _{n \rightarrow \infty} \frac{1}{n} \log \left|E_{n}^{\omega}\right| .
$$

Now let $E$ be a maximal $(\omega, \delta, n)$-separated set in $K^{\omega}$. Then $\bigcup_{x \in E} B_{x}^{\omega}(\delta, n) \supset K^{\omega}$, and $B_{x}^{\omega}(\delta / 2, n), B_{y}^{\omega}(\delta / 2, n)$ are disjoint for any $x, y \in K^{\omega}, x \neq y$. It is clear that any $B_{x}^{\omega}(\delta / 2, n)$ contains a ball of radius $\frac{1}{2} \delta\left(\max _{1 \leq i \leq d} \prod_{j=0}^{n-1} m_{i}\left(\theta^{j} \omega\right)\right)^{-1}$ centered at $x$. By volume considerations, any cylinder $C_{\alpha_{0}, \ldots, \alpha_{n-1}}^{\omega}$ cannot intersect more than $10 \delta^{-d} k_{n}(\omega)$ such balls, where $k_{n}(\omega)$ is the same as in (2.17). Then $|E| \leq$ $10 \delta^{-d} k_{n}(\omega) r_{n}^{\omega}(K)$, and since with probability one $\frac{1}{n} \log k_{n}(\omega) \rightarrow 0$ as $n \rightarrow \infty$, I derive from (4.7) that $h_{\text {top }}^{(r)}(\tau, K) \leq \liminf _{n \rightarrow \infty} \frac{1}{n} \log r_{n}^{\omega}(K)$. In order to obtain the inequality in the other direction, observe that each $(\omega, n)$-cylinder which intersects $K^{\omega}$ intersects also some of the sets $B_{x}^{\omega}(\delta, n), x \in E$. Each such set is contained in a cube with side $2 \delta\left(\min _{1 \leq i \leq d} \prod_{j=0}^{n-1} m_{i}\left(\theta^{j} \omega\right)\right)^{-1}$. By volume considerations such a cube can intersect no more than $(3 \delta)^{d} k_{n}(\omega)(\omega, n)$-cylinders. Thus $r_{n}^{\omega}(K) \leq(3 \delta)^{d} k_{n}(\omega)|E|$, which by (4.7) yields $h_{\text {top }}^{(r)}(\tau, K) \geq \lim \sup _{n \rightarrow \infty} \frac{1}{n} \log r_{n}^{\omega}(K)$, completing the proof of (4.6).

Now I can complete the proof of Theorem 4.1. By (2.13), (2.18), and Lemma 4.2 for any $\delta, \rho>0$ and $P$-a.a. $\omega$ there exists $n_{0}(\omega)$ such that for all $n \geq n_{0}(\omega)$,

$$
\lambda_{\kappa}^{\omega}\left(K^{\omega}, \rho\right) \leq r_{n}^{\omega}(K)\left|C_{\alpha_{0}, \ldots, \alpha_{n-1}}^{\omega}\right| \leq e^{n\left(h_{\text {top }}^{(r)}(\tau, K)+\delta\right)} e^{-n \kappa\left(d \int \log m_{1} d P-\delta\right)},
$$

where $\lambda_{\kappa}^{\omega}$ was defined by $(2.14)$. Let

$$
\kappa>\frac{h_{\text {top }}^{(r)}(\tau, K)}{d \int \log m_{1} d P} \quad \text { and } \quad \delta<\frac{1}{2}\left(\kappa d \int \log m_{1} d P-h_{\text {top }}^{(r)}(\tau, K)\right) .
$$

Then the right hand side of (4.8) tends to zero as $n \rightarrow \infty$, and so in view of Lemma 2.2 I derive that

$$
H D\left(K^{\omega}\right) \leq \frac{h_{\mathrm{top}}^{(r)}(\tau, K)}{\int \log m_{1} d P}
$$

concluding the proof of Theorem 4.1.

4.3. Remark. One obtains the sets $K^{\omega}, \omega \in \Omega$, satisfying (1.10) by choosing, for instance, a measurable family of finite subsets $\Psi(\omega) \subset\left\{l-\mathbf{1}: l \in \mathbb{Z}_{+}^{d}, l \leq m(\omega)\right\}=$ $\mathcal{L}(\omega)$ and setting $K^{\omega}=\left\{x \in \mathbb{T}^{d}: x_{j}(\omega) \in \Psi\left(\theta^{j} \omega\right) \forall j=0,1, \ldots\right\}$. If with positive probability $\Psi(\omega) \neq \mathcal{L}(\omega)$, then for $P$-a.a. $\omega$ the $K^{\omega}$ are proper closed subsets of $\mathbb{T}^{d}$ which can be called random Cantor sets. They are statistically self similar in the sense that if $\alpha_{j} \in \Psi\left(\theta^{j} \omega\right), j=0,1, \ldots, n-1$, then looking at the intersection of $K^{\omega}$ with the cylinder $C_{\alpha_{0}, \ldots, \alpha_{n-1}}^{\omega}$ and rescaling by means of $M\left(\theta^{n-1} \omega\right) \cdots M(\omega)$, where $M(\omega)$ is the same as in (1.2), we obtain $K^{\theta^{n} \omega}$, namely, $f^{n}(\omega)\left(K^{\omega} \cap C_{\alpha_{0}, \ldots, \alpha_{n-1}}^{\omega}\right)=$ 
$K^{\theta^{n} \omega}$, which has the same distribution as $K^{\omega}$. Observe that $h_{\text {top }}^{(r)}(\tau, K)$ for such sets $K^{\omega}$ is equal to $\int \log |\Psi(\omega)| d P(\omega)$. More general family of sets $K^{\omega}$ satisfying (1.10) can be obtained by taking a measurable family of matrices $B(\omega)=\left(b_{k l}(\omega), k, l \in\right.$ $\left.\mathbb{Z}_{+}^{d}, k \leq m(\omega), l \leq m(\theta \omega)\right)$ with 0 and 1 entries and by setting $K^{\omega}=\left\{x \in \mathbb{T}^{d}\right.$ : $\left.b_{x_{i}(\omega)+\mathbf{1}, x_{i+1}(\omega)+\mathbf{1}}=1 \forall i=0,1, \ldots\right\}$. In this case, with probability one,

$$
h_{\mathrm{top}}^{(r)}(\tau, K)=\lim _{n \rightarrow \infty} \frac{1}{n} \log \left\|B\left(\theta^{n-1} \omega\right) \cdots B(\theta \omega) B(\omega)\right\| .
$$

This example is connected with random subshifts of finite type considered in [BG2] and $[\mathrm{KK}]$. A somewhat related situation was studied in [Bed].

It is not difficult to understand that the Hausdorff and Minkowski dimensions (the latter is called also the box dimension, see [Fa]) coincide for the sets $K^{\omega}$ considered in Theorem 4.1. I shall show next that these dimensions will usually be different for such sets if (1.1) is replaced by (3.12) with possibly different $\gamma_{i}$ 's, which in my random set yields the results similar to [Mc] and [KP2]. Thus, assume that $m(\omega)=\left(m_{1}(\omega), \ldots, m_{d}(\omega)\right) \in \mathbb{Z}_{+}^{d}$ satisfies (3.12) with $\gamma_{1} \leq \gamma_{2} \leq \cdots \leq \gamma_{d}$. As in Remark 4.3, choose a measurable family of subsets $\Psi(\omega) \subset\{l-1: l \in$ $\left.\mathbb{Z}_{+}^{d}, l \leq m(\omega)\right\}$ and put $K^{\omega}=\left\{x \in \mathbb{T}^{d}: x_{j}(\omega) \in \Psi\left(\theta^{j} \omega\right)\right\}$. For all $\alpha_{j} \in \mathbb{Z}_{+}^{d}, \alpha_{j} \leq$ $m\left(\theta^{j} \omega\right)-\mathbf{1}, j=0,1, \ldots, n-1$, set

$$
R_{\alpha_{0}, \ldots, \alpha_{n-1}}^{\omega}=\bigcup_{\beta_{0}, \ldots, \beta_{n-1}} C_{\beta_{0}, \ldots, \beta_{n-1}}^{\omega}
$$

where $C_{\beta_{0}, \ldots, \beta_{n-1}}^{\omega}$ are $(\omega, n)$-cylinders as above and the union is taken over all $\beta \in$ $\mathbb{Z}_{+}^{d}, \alpha_{j} \leq m\left(\theta^{j} \omega\right)-\mathbf{1}, j=0, \ldots, n-1$, such that $\beta_{i j}=\alpha_{i j}, \forall j=0,1, \ldots,\left[n \gamma_{1} \gamma_{i}^{-1}\right]-1$ and $\forall i=1, \ldots, d$. Denote also by $R_{n}^{\omega}(x)$ the set $R_{\alpha_{0}, \ldots, \alpha_{n-1}}^{\omega}$ which contains $x \in K^{\omega}$. Clearly, the sets $R_{\alpha_{0}, \ldots, \alpha_{n-1}}^{\omega}$ are either disjoint or coincide, and $K^{\omega}$ is the disjoint union of such sets. These sets are random parallelepipeds which are "approximate cubes" in the same sense as $(\omega, n)$-cylinders were under the condition (1.1), namely that with probability one the ratio of the maximal and the minimal sides is subexponential in $n$ as $n \rightarrow \infty$. Via the same arguments as in Lemma 2.2 this enables me to compute the Hausdorff and Minkowski dimensions of $K^{\omega}$ using only covers by the sets $R_{\alpha_{0}, \ldots, \alpha_{n-1}}^{\omega}$. Following [KP2], introduce functions $Z_{k}^{\omega}(l)=Z_{k}^{\omega}\left(l_{1}, \ldots, l_{k}\right), l=$ $\left(l_{1}, \ldots, l_{d}\right) \in \Psi(\omega)$, successively for $k=d-1, \ldots, 1$ by

$$
Z_{k-1}^{\omega}\left(l_{1}, \ldots, l_{k-1}\right)=\sum_{l_{k}=0}^{m_{k}(\omega)-1}\left(Z_{k}^{\omega}\left(l_{1}, \ldots, l_{k}\right)\right)^{\gamma_{k} \gamma_{k+1}^{-1}},
$$

where $\gamma_{d+1}=\infty$ and $Z_{d}^{\omega}(l)=\chi_{\Psi(\omega)}(l)$ is the indicator of $\Psi(\omega)$.

4.4. Theorem. With probability one

$$
H D\left(K^{\omega}\right)=\gamma_{1}^{-1} \int \log Z_{0}^{\omega} d P(\omega)
$$

Proof. For each $\alpha \in \Psi(\omega)$ set

$$
q_{k, \alpha}^{\omega}=\left(Z_{k}^{\omega}(\alpha)\right)^{\gamma_{k} \gamma_{k+1}^{-1}}\left(Z_{k-1}^{\omega}(\alpha)\right)^{-1}, \quad p_{\alpha}^{\omega}=\prod_{k=1}^{d} q_{k, \alpha},
$$


and

$$
\nu^{\omega}\left(C_{\alpha_{0}, \ldots, \alpha_{n-1}}^{\omega}\right)=\prod_{j=0}^{n-1} p_{\alpha_{j}}^{\theta^{j} \omega} .
$$

It follows from (4.10) that $\sum_{\alpha \in \Psi(\omega)} p_{\alpha}^{\omega}=1$, and so Kolmogorov's extension theorem yields that (4.12) determines a measurable in $\omega$ family of probability measures $\nu^{\omega}$ on $\mathbb{T}^{d}$ such that $\nu^{\omega}\left(K^{\omega}\right)=1$. In the same way as in Section 2 I we conclude that $\nu^{\omega}, \omega \in \Omega$, satisfy $(2.30)$ and the measure $\nu$ given by $d \nu(x, \omega)=d \nu^{\omega}(x) d P(\omega)$ is $\tau$-invariant and ergodic. It follows from (4.10), (4.12), and (4.13) that for all $\alpha_{j} \in \Psi\left(\theta^{j} \omega\right), j=0, \ldots, n-1$,

$$
\nu^{\omega}\left(R_{\alpha_{0}, \ldots, \alpha_{n-1}}^{\omega}\right)=\prod_{k=1}^{d} \prod_{j=0}^{N_{k}(n)} q_{k, \alpha_{j}}^{\theta^{j} \omega},
$$

where $N_{k}(n)=\left[n \gamma_{1} \gamma_{k}^{-1}\right]-1$, and so for each $x \in K^{\omega}$,

$$
\begin{aligned}
\log \nu^{\omega} & \left(R_{n}^{\omega}(x)\right)=\sum_{k=1}^{d} \sum_{j=0}^{N_{k}(n)} \log q_{k, x_{j}(\omega)}^{\theta^{j} \omega} \\
= & \sum_{k=1}^{d} \sum_{j=0}^{N_{k}(n)}\left(\gamma_{k} \gamma_{k+1}^{-1} \log Z_{k}^{\theta^{j} \omega}\left(x_{j}(\omega)\right)-\log Z_{k-1}^{\theta^{j} \omega}\left(x_{j}(\omega)\right)\right) \\
= & -\sum_{j=0}^{n-1} \log Z_{0}^{\theta^{j} \omega}+\sum_{k=1}^{d-1}\left(\gamma_{k} \gamma_{k+1}^{-1} g_{k}^{\omega}\left(x, N_{k}(n)\right)-g_{k}^{\omega}\left(x, N_{k+1}(n)\right)\right),
\end{aligned}
$$

where $g_{k}^{\omega}(x, j)=\sum_{i=0}^{j} \log Z_{k}^{\theta^{i} \omega}\left(x_{i}(\omega)\right)$. By the ergodic theorem, for $\nu$-a.a. $(x, \omega)$,

$$
\lim _{n \rightarrow \infty} \frac{1}{n} g_{k}^{\omega}\left(x, N_{j}(n)\right)=\gamma_{1} \gamma_{j}^{-1} \int \log Z_{k}^{\omega}\left(x_{0}(\omega)\right) d \nu^{\omega}(x) d P(\omega),
$$

and together with (4.15) these yield that, for $\nu$-a.a. $(x, \omega)$,

$$
\lim _{n \rightarrow \infty} \frac{1}{n} \log \nu^{\omega}\left(R_{n}^{\omega}(x)\right)=-\int \log Z_{0}^{\omega} d P(\omega) .
$$

Observe that $P$-a.s., for all $x \in \mathbb{T}^{d}$,

$$
\begin{aligned}
\lim _{n \rightarrow \infty} & \frac{1}{n} \log \operatorname{Leb}\left(R_{n}^{\omega}(x)\right)=\lim _{n \rightarrow \infty}-\frac{1}{n} \sum_{k=1}^{d} \sum_{j=0}^{N_{k}(n)} \log m_{k}\left(\theta^{j} \omega\right) \\
= & -\sum_{k=1}^{d} \gamma_{1} \gamma_{k}^{-1} \int \log m_{k} d P=-\gamma_{1} d .
\end{aligned}
$$

Denote by $K_{0}^{\omega}$ the subset of $x^{\prime}$ 's from $K^{\omega}$ for which (4.17) holds true $P$-a.s. Then arguments similar to Lemma 2.2 enable me to apply Proposition 2.3 to the sets $R_{n}^{\omega}(x)$ in place of $(\omega, n)$-cylinders in Section 2, which together with (2.22) yields

$$
H D\left(K^{\omega}\right) \geq H D\left(K_{0}^{\omega}\right) \geq \gamma_{1}^{-1} \int \log Z_{0}^{\omega} d P(\omega) .
$$

In order to derive the inequality in the other direction I shall need first the following result which improves Lemma 6.1 from [KP2] with essentially the same proof, which I give here for the reader's convenience. 
4.5 Lemma. (i) Let $\varphi_{k}(y), k=1, \ldots, d$, be measurable functions on $(0, \infty)$ satisfying

$$
\max _{1 \leq k \leq d} \sup _{y \geq \delta} y^{-1}\left|\varphi_{k}(y)\right|<\infty
$$

for all $\delta>0$. Then for any numbers $\rho_{k}>0, k=1, \ldots, d$,

$$
\limsup _{y \rightarrow \infty} \sum_{k=1}^{d} y^{-1}\left(\varphi_{k}(y)-\rho_{k}^{-1} \varphi_{k}\left(\rho_{k} y\right)\right) \geq 0 .
$$

(ii) Let $\varphi_{k}(n), k=1, \ldots, d$, be functions on $\mathbb{Z}_{+}$satisfying (4.20) and

$$
\max _{1 \leq k \leq d} \limsup _{n \rightarrow \infty} n^{-1}\left|\varphi_{k}(n)-\varphi_{k}(n-1)\right|=0 ;
$$

then for any numbers $\rho_{k}>0, k=1, \ldots, d$,

$$
\limsup _{n \rightarrow \infty} \sum_{k=1}^{d} n^{-1}\left(\varphi_{k}(n)-\rho_{k}^{-1} \varphi_{k}\left(\left[\rho_{k} n\right]\right)\right) \geq 0
$$

Proof. Set $\psi_{k}(z)=e^{-z} \varphi_{k}\left(e^{z}\right), k=1, \ldots, d$. Clearly, for any $u>0$,

$$
\begin{aligned}
& \left|\int_{0}^{u} \sum_{k=1}^{d}\left(\psi_{k}(z)-\psi_{k}\left(z+\log \rho_{k}\right)\right) d z\right| \\
& \quad \leq \sum_{k=1}^{d}\left(\left|\int_{0}^{\log \rho_{k}} \psi_{k}(z) d z\right|+\left|\int_{u}^{u+\log \rho_{k}} \psi_{k}(z) d z\right|\right) .
\end{aligned}
$$

By $(4.20) \psi_{k}(z)$ is bounded on any interval $[a, \infty), a>-\infty$, and so the right hand side of (4.24) is a bounded function of $u$. It follows that

$$
\limsup _{z \rightarrow \infty} \sum_{k=1}^{d}\left(\psi_{k}(z)-\psi_{k}\left(z+\log \rho_{k}\right)\right) \geq 0,
$$

since otherwise the left hand side of (4.24) would grow at least linearly in $u$ for $u$ big enough. Set $y=e^{z}$; then $\psi_{k}(z)=y^{-1} \varphi_{k}(y)$, and $\psi_{k}\left(z+\log \rho_{k}\right)=\rho_{k}^{-1} y^{-1} \varphi_{k}\left(\rho_{k} y\right)$, and (4.21) follows from (4.25). If the $\varphi_{k}$ 's are defined only on $\mathbb{Z}_{+}$and satisfy (4.20), I extend them linearly inside each interval $[n, n+1]$, and (4.23) will follow from (4.20)-(4.22).

Now I can complete the proof of Theorem 4.4. Observe that for each $k=1, \ldots, d$ and $\alpha \in \Psi(\omega)$,

$$
Z_{k}^{\omega}(\alpha) \leq \prod_{i=1}^{d} m_{i}(\omega)
$$

Since

$$
\lim _{n \rightarrow \infty} n^{-1}\left(N_{k+1}(n)-N_{k}\left(\left[\gamma_{k} \gamma_{k+1}^{-1} n\right]\right)\right)=0,
$$


then by (3.12), (4.26), the definition of $q_{k}^{\omega}(x, j)$, and by the ergodic theorem, for $P$-a.a. $\omega$ and for each $x \in K^{\omega}$,

$$
\lim _{n \rightarrow \infty} n^{-1}\left(g^{\omega}\left(x, N_{k}\left(\left[\gamma_{k} \gamma_{k+1}^{-1} n\right]\right)\right)-g^{\omega}\left(x, N_{k+1}(n)\right)\right)=0 .
$$

Set $\varphi_{k}^{\omega}(x, n)=\gamma_{k} \gamma_{k+1}^{-1} g^{\omega}\left(x, N_{k}(n)\right) ;$ then

$$
g^{\omega}\left(x, N_{k}\left(\left[\gamma_{k} \gamma_{k+1}^{-1} n\right]\right)\right)=\gamma_{k+1} \gamma_{k}^{-1} \varphi_{k}^{\omega}\left(\left[\gamma_{k} \gamma_{k+1}^{-1} n\right]\right) .
$$

By (4.26) and the ergodic theorem, for $P$-a.a. $\omega$,

$$
\begin{aligned}
\limsup _{n \rightarrow \infty} n^{-1} \sup _{x \in K^{\omega}}\left|\varphi_{k}^{\omega}(x, n)\right| & \leq \gamma_{k} \gamma_{k+1}^{-1} \limsup _{n \rightarrow \infty} n^{-1} \sum_{j=0}^{N_{k}(n)} \sum_{i=1}^{d} \log m_{i}\left(\theta^{j} \omega\right) \\
& =\gamma_{1} \gamma_{k+1}^{-1} \sum_{i=1}^{d} \gamma_{i}<\infty
\end{aligned}
$$

and

$$
\begin{aligned}
& \limsup _{n \rightarrow \infty} n^{-1} \sup _{x \in K^{\omega}}\left|\varphi_{k}^{\omega}(x, n)-\varphi_{k}^{\omega}(x, n-1)\right| \\
& \leq \gamma_{k} \gamma_{k+1}^{-1} \limsup _{n \rightarrow \infty} n^{-1} \sum_{i=1}^{d} \log m_{i}\left(\theta^{N_{k}(n)} \omega\right)=0,
\end{aligned}
$$

which implies both (4.20) and (4.22). Thus I can apply Lemma 4.5 with $\varphi_{k}(n)=$ $\varphi_{k}^{\omega}(x, n)$ and $\rho_{k}=\gamma_{k} \gamma_{k+1}^{-1}$, which in view of (4.15), (4.27)-(4.29), and the ergodic theorem yields that, for $P$-a.a. $\omega$ and any $x \in K^{\omega}$,

$$
\limsup _{n \rightarrow \infty} \frac{1}{n} \log \nu^{\omega}\left(R_{n}^{\omega}(x)\right) \geq-\int \log Z_{0}^{\omega} d P(\omega) .
$$

This together with (4.18) implies that, for $P$-a.a. $\omega$ and each $x \in K^{\omega}$,

$$
\liminf _{n \rightarrow \infty} \frac{\log \nu^{\omega}\left(R_{n}^{\omega}(x)\right)}{\log \left(\operatorname{Leb} R_{n}^{\omega}(x)\right)} \leq \gamma_{1}^{-1} d^{-1} \int \log Z_{0}^{\omega} d P(\omega) .
$$

Finally, by (2.23) and (2.26) I conclude from (4.30) that with probability one

$$
H D\left(K^{\omega}\right) \leq \gamma_{1}^{-1} \int \log Z_{0}^{\omega} d P(\omega)
$$

which together with (4.19) completes the proof of Theorem 4.4.

Concerning the Minkowski dimension of the $K^{\omega}$ 's, one has the following.

4.6. Theorem. (i) With probability one

$$
M D\left(K^{\omega}\right)=\sum_{k=1}^{d}\left(\gamma_{k}^{-1}-\gamma_{k+1}^{-1}\right) \int \log \left|\pi_{k}(\Psi(\omega))\right| d P(\omega)
$$


where $\pi_{k}$ is the projection to the first $k$ coordinates and $\gamma_{d+1}=\infty$.

(ii) The equality $M D\left(K^{\omega}\right)=H D\left(K^{\omega}\right)$ holds true with probability one if and only if for each $k=1, \ldots, d-1$ the cardinalities of the preimages under $\pi_{k}$ are the same for all elements of $\pi_{k}(\Psi(\omega))$ and P-a.a. $\omega$.

Proof. It is not difficult to understand via arguments similar to Lemmas 2.2 and 4.2 that with probability one

$$
M D\left(K^{\omega}\right)=\gamma_{1}^{-1} \lim _{n \rightarrow \infty} \frac{1}{n} \log r_{n}^{\omega}(K)
$$

where $r_{n}^{\omega}(K)$ is the number of different sets $R_{\alpha_{0}, \ldots, \alpha_{n-1}}^{\omega}$ which intersect $K^{\omega}$. From the definition of $K^{\omega}$ it follows that

$$
r_{n}^{\omega}(K)=\prod_{k=1}^{d} \prod_{j=N_{k+1}(n)+1}^{N_{k}(n)}\left|\pi_{k}\left(\Psi\left(\theta^{j} \omega\right)\right)\right|
$$

where, by convention, $N_{d+1}(n)=0$. Now (4.32) and (4.33) together with the ergodic theorem yield (4.31). The assertion (ii) is obtained similarly to [KP2] by comparing (4.11) and (4.31).

4.7. Remark. Similarly to [KP2] I can obtain in the case $d=2$ also the Hausdorff dimension of sets $K^{\omega}, \omega \in \Omega$, determined by random subshifts of finite type in place of the restriction on each $x_{j}(\omega)$ separately (see Remark 4.3 ). Also it is not difficult to obtain random (relativized) versions of the Ledrappier-Young formula from [KP2]. Combining methods of [KP1] and of the present paper, one can obtain also the Hausdorff dimension of the sets $\left(K^{\omega}+t\right) \cap K^{\omega}$, where $t \in \mathbb{T}^{d}$ with $K^{\omega} \subset \mathbb{T}^{d}$ satisfying (1.10). In particular, similarly to Theorem 1.2 in [KP1] I derive that if $d=1$ and $K_{\Psi}^{\omega}=\left\{x \in[0,1): x_{j}(\omega) \in \Psi\left(\theta^{j} \omega\right) \forall j=0,1, \ldots\right\}$ are random Cantor sets, then with probability one

$$
H D\left(\left(K_{\Psi_{1}}^{\omega}+t\right) \cap K_{\Psi_{2}}^{\omega}\right)=\frac{\lambda}{\int \log m d P}
$$

for Leb a.a. $t \in[0,1)$, where $\lambda=\lim _{n \rightarrow \infty} \frac{1}{n} \log \left\|A_{\phi \circ \tau^{n}(t, \omega)}^{\theta^{n} \omega} \cdots A_{\phi \circ \tau(t, \omega)}^{\theta \omega} A_{\phi(t, \omega)}^{\omega}\right\|$ with $\phi(t, \omega)=t_{0}(\omega)$ and $A_{0}^{\omega}, A_{1}^{\omega}, \ldots, A_{m(\omega)-1}^{\omega}$ are two-by-two matrices given by $A_{l}^{\omega}(i, j)=\left|\left(\Psi_{1}(\omega)+i+l\right) \cap\left(\Psi_{2}(\omega)+j m(\omega)\right)\right|$ for $i, j=0,1$. The above limit exists and is constant with probability one for Leb a.a. $t \in[0,1)$, since Leb $\times P$ is an ergodic $\tau$-invariant probability measure on $[0,1) \times \Omega$. Similarly to Theorem 3.1 in [KP1], under additional assumptions one can obtain the above Hausdorff dimension in a more explicit form. Suppose, for instance, that the difference set $\Psi(\omega)=\Psi_{2}(\omega)-\Psi_{1}(\omega) \subset \mathbb{Z}$ is contained in an arithmetic progression of length $m(\omega)$ and $|\Psi(\omega)|=m(\omega)$. Then with probability one

$$
H D\left(\left(K_{\Psi_{1}}^{\omega}+t\right) \cap K_{\Psi_{2}}^{\omega}\right)=\frac{1}{\int \log m d P} \int \frac{1}{m(\omega)} \sum_{i \in \Psi(\omega)} \log M_{i}^{\omega} d P(\omega)
$$

for Leb a.a. $t$ from the interval $K_{\Psi}^{\omega}=K_{\Psi_{2}}^{\omega}-K_{\Psi_{1}}^{\omega}$, where $M_{i}^{\omega}=\left|\left(\Psi_{1}(\omega)+i\right) \cap \Psi_{2}(\omega)\right|$.

Next, I shall exhibit a version of the Bowen-Ruelle formula for the Hausdorff dimensions of repellers of random conformal maps. Let $(\Omega, P)$ and $\theta$ be as before, 
$M$ be a $C^{2}$ locally compact Riemannian manifold, and $f(\omega), \omega \in \Omega$, be a measurable family of $C^{2}$ maps of $M$ such that there exist a compact set $M_{0} \subset M$ with nonempty interior $\operatorname{int} M_{0}$ and a function $\lambda_{\omega}(x)$ satisfying:

$$
D_{x} f(\omega)=\lambda_{\omega}(x) I_{x}^{\omega}, \quad \forall x \in M_{0}, \omega \in \Omega,
$$

where $D_{x} f(\omega)$ is the differential of $f(\omega)$ at $x$ and $I_{x}^{\omega}$ is an isometry of the tangent space $T_{x} M$ onto $T_{f(\omega)} M$.

(ii) With probability one, $\inf _{x \in M_{0}} \lambda_{\omega}(x)>1$.

(iii) If $\gamma_{0}(\omega)=\sup _{x \in M_{0}} \log \lambda_{\omega}(x)$ and $\gamma_{1}(\omega)=\sup _{x \in M_{0}}\left|\log \left\|\frac{d \lambda_{\omega}(x)}{d x}\right\|\right|$ then $\int\left(\gamma_{0}+\gamma_{1}\right) d P<\infty$.

I assume also the mixing condition saying that for any open set $U \subset M_{0}$ there exists $N=N_{\omega}$ such that $f^{N}(\omega) U \supset M_{0}$, where $f^{N}(\omega)=f\left(\theta^{N} \omega\right) \circ \cdots \circ f(\theta \omega) f(\omega)$. As an example of maps satisfying the above conditions one may keep in mind algebraic endomorphisms of the torus $\mathbb{T}^{d}$ given by integer valued matrices $L=\left(l_{i j}\right)$ such that $\sum_{j=1}^{d} l_{i j} l_{k j}=\delta_{i k} \lambda_{L}^{2}$ for some $\lambda_{L}>1$ independent of $i$, where $\delta_{i k}=1$ if $i=k$ and $=0$, otherwise. Then $\lambda_{L}^{-1} L$ is an orthogonal matrix, and the conditions above are satisfied for $f(\omega)$ taken out of this family of endomorphisms with $\lambda_{\omega}(x)$ depending on $\omega$ but not on $x$.

Now let $K^{\omega}, \omega \in \Omega$, be a measurable family of compact sets satisfying (1.10) and $K^{\omega} \subset M_{1}, \forall \omega \in \Omega$ for some compact set $M_{1} \subset \operatorname{int} M_{0}$. I shall use again the metrics $\rho_{n}^{\omega}(x, y)$, the sets $B_{\delta}^{\omega}(x, n)$, and the notion of $(\omega, \delta, n)$-separated sets defined in the proof of Lemma 4.2 with $\mathbb{T}^{d}$ replaced by $M$. Let $\psi_{\omega}(x), \omega \in \Omega$, be a family of continuous functions on $M_{1}$ such that $\int \sup _{x \in M_{1}}\left|\psi_{\omega}(x)\right| d P(\omega)<\infty$. Let $E_{n}^{\omega} \subset K^{\omega}$ be any family of maximal $(\omega, \delta, n)$-separated sets in $K^{\omega}$; then the relativized topological pressure $Q_{K}^{(r)}(\psi)$ of $\psi=\psi_{\omega}(x)$ on $K=\left\{(x, \omega): x \in K^{\omega}\right\}$ can be obtained for $P$-a.a. $\omega$ by

$$
Q_{K}^{(r)}(\psi)=\limsup _{\delta \rightarrow 0} \limsup _{n \rightarrow \infty} \frac{1}{n} \log \sum_{x \in E_{n}^{\omega}} \exp \left(S_{n} \psi(x, \omega)\right)
$$

where $S_{n} \psi(x, \omega)=\sum_{j=0}^{n-1} \psi_{\theta^{j} \omega}\left(f^{j}(\omega) x\right)$. Note that if $\psi$ is independent of $x$ on $K$, then

$$
Q_{K}^{(r)}(\psi)=Q_{K}^{(r)}(0)+\lim _{n \rightarrow \infty} \frac{1}{n} \sum_{i=0}^{n-1} \psi_{\theta^{i} \omega}=h_{\mathrm{top}}^{(r)}(\tau, K)+\int \psi_{\omega} d P(\omega)
$$

where, again, $\tau$ is the skew product transformation and $h_{\text {top }}^{(r)}(\tau, K)$ is the relativized topological entropy of $\tau$ on $K$. One has also the following relativized variational principle (see $[\mathrm{Bo}]$ ):

$$
Q_{K}^{(r)}(\psi)=\sup _{\mu}\left(\int \psi d \mu+h_{\mu}^{(r)}(\tau)\right)
$$

where, again, $h_{\mu}^{(r)}(\tau)$ denotes the relativized entropy of $\tau$ with respect to $\mu$, and the supremum is taken over the set of all $\tau$-invariant probability measures on $K$ whose marginal on $\Omega$ is $P$. 
4.8. Theorem. Let $\varphi_{\omega}(x)=-\log \lambda_{\omega}(x)$. Then, under the assumptions above, for P-a.a. $\omega$,

$$
H D\left(K^{\omega}\right)=t_{0} \quad \text { if } Q_{K}^{(r)}\left(t_{0} \varphi\right)=0 .
$$

If $\lambda_{\omega}(x)$, and so $\varphi_{\omega}(x)$, are independent of $x$ on $K$, then

$$
H D\left(K^{\omega}\right)=\frac{h_{\mathrm{top}}^{(r)}(\tau, K)}{\int \log \lambda_{\omega} d P(\omega)} .
$$

Proof. First, observe that (4.38) follows immediately from (4.34)-(4.37) if $\lambda_{\omega}(x)$ does not depend on $x$. Now let $E_{n}^{\omega}$ be a family of maximal $(\omega, \delta, n)$-separated sets in $K^{\omega}, \omega \in \Omega$. In the same way as in Section 3 of $[\mathrm{KK}]$ via Proposition 2.1 there, it follows from (i)-(iii) that for any $\varepsilon>0$ there exists a measurable set $\Omega_{\varepsilon} \subset \Omega$ with $P\left(\Omega \backslash \Omega_{\varepsilon}\right)<\varepsilon$ and random variables $R_{\varepsilon, \delta}(\omega)<\infty$ such that for any $\delta \leq \delta_{0}(\omega)$, $x \in K^{\omega}, P$-a.a. $\omega$, and $n$ satisfying $\theta^{n} \omega \in \Omega_{\varepsilon}$,

$$
B_{R_{\varepsilon, \delta}(\omega) \Lambda_{n}^{\omega}(x)}(x) \supset B_{\delta}^{\omega}(x, n) \supset B_{R_{\varepsilon, \delta}^{-1}(\omega) \Lambda_{n}^{\omega}(x)}(x),
$$

where $B_{r}(x)$ denotes the ball centered at $x$ with radius $r$ and

$$
\Lambda_{n}^{\omega}(x)=\left(\prod_{j=0}^{n-1} \lambda_{\theta^{j} \omega}\left(f^{j}(\omega) x\right)\right)^{-1}
$$

Then

$$
\sum_{x \in E_{n}^{\omega}} \exp \left(t S_{n} \varphi(x, \omega)\right)=\sum_{x \in E_{n}^{\omega}}\left(\Lambda_{n}^{\omega}(x)\right)^{t} .
$$

For $t=t_{0}$ the expression in the left hand side of (4.40) is subexponential in $n$, and for $t>t_{0}$ it tends to zero as $n \rightarrow \infty$ in view of (ii). Since

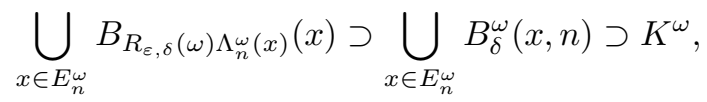

I derive from here that

$$
H D\left(K^{\omega}\right) \leq t_{0} .
$$

In order to prove the inequality in the other direction one can employ a relativized version of the Brin-Katok local entropy formula (see [BK]) saying that for any $\tau$ invariant ergodic probability measure $\mu$ on $K$ such that $d \mu(x, \omega)=d \mu^{\omega}(x) d P(\omega)$ and $\mu$-a.a. $(x, \omega)$,

$$
\lim _{n \rightarrow \infty} \frac{1}{n} \log \mu^{\omega}\left(B_{\delta}^{\omega}(x, n)\right)=-h_{\mu}^{(r)}(\tau) .
$$


Let $\mu$ be the equilibrium state corresponding to the function $t_{0} \varphi$ (see [Ki1], [KK]). Then by (4.36),

$$
h_{\mu}^{(r)}(\tau)=-t_{0} \int \varphi d \mu
$$

Since $\mu$ is ergodic (see $[\mathrm{KK}]$ ), then for $\mu$-a.a. $(x, \omega)$,

$$
\lim _{n \rightarrow \infty} \frac{1}{n} \log \Lambda_{n}^{\omega}(x)=-\int \log \lambda_{\omega}(x) d \mu(x, \omega)<0
$$

and, in particular,

$$
\lim _{n \rightarrow \infty} \frac{\log \Lambda_{n+1}^{\omega}(x)}{\log \Lambda_{n}^{\omega}(x)}=1
$$

Observe also that if $n_{j}^{(\varepsilon)}(\omega), i=1,2, \ldots$, are successive integers for which $\theta^{n_{j}^{(\varepsilon)}(\omega)} \omega \in$ $\Omega_{\varepsilon}, \varepsilon \in(0,1)$, then by the ergodic theorem, for $P$-a.a. $\omega$,

$$
\lim _{j \rightarrow \infty} \frac{n_{j+1}^{(\varepsilon)}(\omega)}{n_{j}^{(\varepsilon)}(\omega)}=1 .
$$

This together with (4.39),(4.42)-(4.45), and the Fubini theorem yields that, with probability one for $\mu^{\omega}$-a.a. $x$,

$$
\lim _{\delta \rightarrow 0} \frac{\log \mu^{\omega}\left(B_{\delta}(x)\right)}{\log \delta}=t_{0}
$$

Let $K_{0}^{\omega} \subset K^{\omega}$ be the set of $x$ for which (4.47) holds true. Then from Proposition 2.1 in [Yo] (which is one of the general versions of Proposition 2.3 in Section 2) I obtain that $H D\left(K^{\omega}\right) \geq H D\left(K_{0}^{\omega}\right)=t_{0}$, which together with (4.41) completes the proof of Theorem 4.8.

4.9. Remark. Modifying the proof in $[\mathrm{Ru}]$ one can obtain that for $P$-a.s. $\omega$ the disintegrations $\mu^{\omega}$ of $\mu$ are equivalent to $t_{0}$-Hausdorff measures on $K^{\omega}, \omega \in \Omega$.

4.10. Remark. It is also possible to generalize to the case of random diffeomorphisms Young's formula [Yo] which expresses dimensions of invariant measures of diffeomorphisms of surfaces via entropies and Lyapunov exponents. Other dimensions considered in [Yo] such as Rényi and Lyapunov dimension, as well as the correlation dimension, can be also defined and studied in the case of random diffeomorphisms.

4.11. Remark. One can obtain the lower bound in the proof of Theorem 4.8 by constructing a random Markov partition (see [BG1]) on a small neighborhood of the set $M_{1}$ and applying the relativized the Shannon-McMillan-Breiman theorem (see [Bo]) in place of the relativized Brin-Katok local entropy formula. The application of Shannon-McMillan-Breiman theorem is possible since similarly to the deterministic case the boundary of a Markov partition has zero measure with respect to any equilibrium state corresponding to a "good" function (see $[\mathrm{KK}]$ ), the 
argument being completed by some general version (see [Bi3]) of Proposition 2.3 above. Constructing Markov partitions and passing to symbolic representations (random subshifts of finite type, see [BG2]) also enables one to obtain a lot of nontrivial sets $K^{\omega}$ satisfying (1.10) taking sequences which do not contain prescribed symbols (cf. Remark 4.3).

4.12. Remark. One can study an expanding map by means of its inverse branches, which form an iterated function system consisting of contractions. Similarly, random expanding maps can be studied via random iterated function systems defined as follows. Let $\Omega, P, \theta$ be as before, $(X, \rho)$ be a complete metric space, $\mathcal{S}=\left(f_{1}, f_{2}, \ldots\right)$ be a countable set of contractions of $X$, and $S(\omega)$ be a measurable family of finite subsets of $\mathbb{Z}_{+}$. Set $s(\omega)=\max \left\{s_{f_{i}}: i \in S(\omega)\right\}$, where $s_{f}$ is the contraction factor of $f$ and $m(\omega)=|S(\omega)|$. Put $A^{\omega}=\left\{a=\left(a_{0}, a_{1}, \ldots\right): a_{i} \in S\left(\theta^{i} \omega\right)\right\}$ and consider iterations $f_{a}^{n}=f_{a_{0}} \circ f_{a_{1}} \circ \cdots \circ f_{a_{n-1}}$. Suppose that

$$
\int \log s(\omega) d P(\omega)<0 \quad \text { and } \quad \int \log m(\omega) d P(\omega)<\infty .
$$

The first condition in (4.48) implies that with probability one for any $x \in X$ and $a \in A^{\omega}$ the limit $\lim _{n \rightarrow \infty} f_{a}^{n} x=x_{a}(\omega)$ exists and the set $K^{\omega}=\left\{x_{a}(\omega): a \in\right.$ $\left.A^{\omega}\right\}$ satisties $F(\omega) K^{\theta \omega}=K^{\omega}$, where $F(\omega) K=\bigcup_{\alpha \in S(\omega)} f_{\alpha} K$. This enables me to construct random repellers for random expanding (in particularly, conformal) maps. Namely, given random expanding maps $f(\omega), \omega \in \Omega$, I choose the set of indices $S(\omega)$ so that it corresponds to inverse branches of $f(\omega)$, which are contracting maps. For instance, if $f(\omega)$ 's are taken out of a family of rational maps of the Riemannian sphere with hyperbolic Julia sets close to each other, then all inverse branches will be contractions in some fixed compact set. Another important feature of random iterated function systems consisting of contractions is the possibility to employ the Ruelle-Perron-Frobenius operator, which yields some of the results from [Ki2] and $[\mathrm{KK}]$ for this more general setup. As an example, let us see how the random Cantor sets from Remark 4.3 can be obtained via random iterated function systems. Let $\mathrm{d}=1$ and $\Psi(\omega) \subset\{0,1, \ldots, m(\omega)-1\}$. Set

and

$$
\mathcal{S}=\left\{f: f x=\frac{x}{m}+\frac{k-1}{m} ; m, k \in \mathbb{Z}_{+}, k \leq m\right\}
$$

$$
S(\omega)=\left\{k \in \Psi(\omega): f_{k} x=\frac{x}{m(\omega)}+\frac{k}{m(\omega)}\right\} .
$$

Then $K^{\omega}=\left\{x \in[0,1): x_{j}(\omega) \in \Psi\left(\theta^{j} \omega\right)\right\}$ can be obtained also as the set of limits $\lim _{n \rightarrow \infty} f_{a}^{n} x$ for $a \in A^{\omega}$. One can construct in this way some random von Koch type curves and other random fractals.

4.13. Remark. Let $f(\omega), \omega \in \Omega$, be piecewise linear maps of $[0,1]$ onto itself such that there exist points $0=z_{0}(\omega)<z_{1}(\omega)<\ldots<z_{m(\omega)-1}<z_{m(\omega)}=1$ with $f(\omega)\left[z_{l}(\omega), z_{l+1}(\omega)\right]=[0,1] \forall l=0, \ldots, m(\omega)-1$. If $\left|z_{l+1}(\omega)-z_{l}(\omega)\right|=\frac{1}{m(\omega)} \forall l=$ $0,1, \ldots, m(\omega)-1$, then we come back to the previous setup with $d=1$. Suppose that

$$
\begin{aligned}
-\infty & <\int \min _{0 \leq l<m(\omega)} \log \left|z_{l+1}(\omega)-z_{l}(\omega)\right| d P(\omega) \\
& \leq \int \max _{0 \leq l<m(\omega)} \log \left|z_{l+1}(\omega)-z_{l}(\omega)\right| d P(\omega)<0 .
\end{aligned}
$$


The maps $f(\omega)$ are conformal (as any smooth one dimensional map) but, in general, the right and the left derivatives of $f(\omega) x$ in $x$ do not coincide at $z_{l}(\omega), l=$ $1, \ldots, m(\omega)-1$. Still one can adapt Theorem 4.8 to this situation (adjusting the method from [Wa] to random transformations similarly to [Ki2] and [KK]) and obtain (4.37) for Hausdorff dimensions of sets $K^{\omega}$ satisfying (1.10), but I do not know an explicit formula such as (4.1) for the Hausdorff dimensions of the corresponding random Cantor sets (for some related results see Section 15.1 in $[\mathrm{Fa}]$ ). Next, I can assign to each $x \in[0,1)$ a sequence $x_{0}(\omega), x_{1}(\omega), \ldots$ (where $x_{j}(\omega)=$ $\left.l-1, l=1, \ldots, m\left(\theta^{j} \omega\right)\right)$, if $f^{j}(\omega) x \in\left[z_{l-1}\left(\theta^{j} \omega\right), z_{l}\left(\theta^{j} \omega\right)\right)$, where, as before, $f^{j}(\omega)=$ $f\left(\theta^{j-1} \omega\right) \circ \cdots \circ f(\omega)$. I can define again $N_{k l}^{\omega}(x, n), N_{l}^{\omega}(x, n), U_{r}^{\omega}$, and $V_{A}^{\omega}$ as in the Introduction and in Section 2. The cylinder sets $C_{n}^{\omega}(x)$ and the random Bernoulli measures $\nu_{p_{A}}^{\omega}$ can be defined as in Section 2. It follows that for any $x \in V_{A}^{\omega}$,

$$
\lim _{n \rightarrow \infty}-\frac{1}{n} \log \nu_{p_{A}}^{\omega}\left(C_{n}^{\omega}(x)\right)=-\sum_{k} q_{k} \sum_{l \leq k} a_{k l} \log a_{k l} .
$$

On the other hand,

$$
\operatorname{Leb}\left(C_{n}^{\omega}(x)\right)=\prod_{j=0}^{n-1} \lambda \circ \tau^{j}(x, \omega),
$$

where $\lambda(x, \omega)=\left|z_{l}(\omega)-z_{l-1}(\omega)\right|$ if $x \in\left[z_{l-1}(\omega), z_{l}(\omega)\right)$, which is more difficult to estimate. Since $\nu_{p_{A}}$ is ergodic, I obtain that with probability one, for $\nu_{p_{A}}^{\omega}$-a.a. $x \in V_{A}^{\omega}$,

$$
\begin{aligned}
\lim _{n \rightarrow \infty} & \frac{1}{n} \log \operatorname{Leb}\left(C_{n}^{\omega}(x)\right)=\int \log \lambda d \nu_{p_{A}} \\
= & \sum_{k, l ; l \leq k} \int_{\Omega_{k}} a_{k l} \log \left|z_{l}(\omega)-z_{l-1}(\omega)\right| d P(\omega) \stackrel{\text { def }}{=} \gamma
\end{aligned}
$$

where $\Omega_{k}=\{\omega: m(\omega)=k\}$. This gives only the lower bound

$$
\gamma^{-1} \sum_{k} q_{k} \sum_{l \leq k} a_{k l} \log a_{k l}
$$

for $H D\left(V_{A}^{\omega}\right)$. It is not clear what is the appropriate upper bound for $H D\left(V_{A}^{\omega}\right)$ and whether one can obtain an explicit formula for this Hausdorff dimension in our circumstances. Observe that in the nonrandom case it is easy, since then $\operatorname{Leb}\left(C_{n}(x)\right)=\prod_{l=1}^{m} \lambda_{l}^{N_{l}(x)}$, where $\lambda_{l}=\left|z_{l}-z_{l-1}\right|$, and so for any $x \in V_{A}$,

$$
\lim _{n \rightarrow \infty}-\frac{1}{n} \log \operatorname{Leb}\left(C_{n}(x)\right)=-\sum_{l=1}^{m} r_{l} \log \lambda_{l}
$$

which yields

$$
H D\left(U_{r}\right)=\frac{\sum_{l} r_{l} \log r_{l}}{\sum_{l} r_{l} \log \lambda_{l}}
$$

Still, there is a natural generalization which can be dealt with. Suppose that $\tilde{m}(\omega)$ is another $\mathbb{Z}_{+}$-valued random variable satisfying $\int \log \tilde{m} d P<\infty$. Assume that 
$\lambda(x, \omega)=\lambda_{\tilde{k} l}$ if $x \in\left[z_{l-1}(\omega), z_{l}(\omega)\right)$ and $\tilde{m}(\omega)=\tilde{k}$, i.e. the lengths $\left|z_{l}(\omega)-z_{l-1}(\omega)\right|$ depend only on $l$ and $\tilde{m}(\omega)$. Denote by $N_{\tilde{k} k l}^{\omega}(x, n)$ the number of $j$ 's between 0 and $n-1$ such that $m\left(\theta^{j} \omega\right)=k, \tilde{m}\left(\theta^{j} \omega\right)=\tilde{k}$, and $x_{j}(\omega)=l-1$. Let $B=\left(b_{\tilde{k} k l}, \tilde{k}, k, l \in\right.$ $\left.\mathbb{Z}_{+}, l \leq k\right)$, where $b_{\tilde{k} k l} \geq 0, \sum_{l \leq k} b_{\tilde{k} k l}=1$, and set

$$
W_{B}^{\omega}=\left\{x \in[0,1): \lim _{n \rightarrow \infty} \frac{1}{n} N_{\tilde{k} k l}^{\omega}(x, n)=u_{\tilde{k} k} b_{\tilde{k} k l} \forall \tilde{k}, k, l \in \mathbb{Z}_{+}\right\}
$$

where $u_{\tilde{k}, k}=P\{m=k, \tilde{m}=\tilde{k}\}$. Set $p_{l}^{\omega}=b_{\tilde{k} k l}$ if $m(\omega)=k$ and $\tilde{m}(\omega)=\tilde{k}$, and denote by $\nu_{p_{B}}^{\omega}$ the unique measure satisfying (2.29). Then for any $x \in W_{B}^{\omega}$, with probability one,

$$
\lim _{n \rightarrow \infty}-\frac{1}{n} \log \nu_{p_{B}}^{\omega}\left(C_{n}^{\omega}(x)\right)=-\sum_{\tilde{k}, k} u_{\tilde{k}, k} \sum_{l \leq k} b_{\tilde{k} k l} \log b_{\tilde{k} k l}
$$

and

$$
\lim _{n \rightarrow \infty}-\frac{1}{n} \operatorname{Leb}\left(C_{n}^{\omega}(x)\right)=-\sum_{\tilde{k}, k} u_{\tilde{k}, k} \sum_{l \leq k} b_{\tilde{k} k l} \log \lambda_{\tilde{k} l} .
$$

Applying a slight modification of Lemma 2.2 (cf. Theorem 1 from [Bi3]), I derive from here that

$$
H D\left(W_{B}^{\omega}\right)=\frac{\sum_{\tilde{k}, k} u_{\tilde{k}, k} \sum_{l \leq k} b_{\tilde{k} k l} \log b_{\tilde{k} k l}}{\sum_{\tilde{k}, k} u_{\tilde{k}, k} \sum_{l \leq k} b_{\tilde{k} k l} \log \lambda_{\tilde{k} l}}
$$

Let $\mathcal{B}_{u, q, A}$ be the set of $B=\left(b_{\tilde{k}, k, l}\right)$ as above such that $\sum_{\tilde{k}} u_{\tilde{k} k} b_{\tilde{k} k l}=q_{k} a_{k l}$ with $q_{k}$ and $A=\left(a_{k l}\right)$ as before. Then

$$
H D\left(V_{A}^{\omega}\right)=\sup _{B \in \mathcal{B}_{u, q, A}} H D\left(W_{B}^{\omega}\right) \text { and } H D\left(U_{r}\right)=\sup _{A \in \mathcal{A}_{q r}} H D\left(V_{A}^{\omega}\right) .
$$

4.14. Remark. Random subshifts considered in [BG2], and [KK] together with the corresponding relativized thermodynamic formalism are the natural framework for general models of random graph directed constructions (see $[\mathrm{Ol}]$ and references there) and random geometric (Moran) constructions (see [PW]) of a different type than considered before. Let $\sigma$ be the shift on the space $\mathcal{X}=\overline{\mathbb{Z}}_{+}^{\mathbb{Z}_{+}}$of sequences $x=\left(x_{i} ; i=1,2, \ldots ; x_{i} \in \overline{\mathbb{Z}}_{+}\right)$considered with the product topology, where $\overline{\mathbb{Z}}_{+}$is the one point compactification of $\mathbb{Z}_{+}$. A random subshift of $(\mathcal{X}, \sigma)$ is a collection of a probability space $(\Omega, \mathcal{F}, P)$ together with a $\mathbb{Z}_{+}$-valued random variable $m(\omega)$ satisfying $\int \log m d P<\infty$, a $P$-preserving transformation $\theta$ of $\Omega$, and a measurable family of compact subsets $X^{\omega} \subset \mathcal{X}$ such that $\sigma X^{\omega}=X^{\theta \omega}$ and if $x=\left(x_{i}\right) \in X^{\omega}$ then $x_{i} \in\{1,2, \ldots, m(\omega)\}$. If there exists a measurable family of $m(\omega) \times m(\theta \omega)$ matrices $B(\omega)$ with entries $b_{k l}(\omega), k=1, \ldots, m(\omega), l=1, \ldots, m(\theta \omega)$, equal to 0 or 1 and such that $X^{\omega}=\left\{x=\left(x_{i}\right): b_{x_{i} x_{i+1}}\left(\theta^{i} \omega\right)=1\right\}$, then the random subshift is said to be of finite type. For any $\alpha=\left(\alpha_{i}\right) \in X^{\omega}$ set $\alpha^{(n)}=\left(\alpha_{1}, \ldots, \alpha_{n}\right)$ and $C_{\alpha^{(n)}}^{\omega}=\left\{x=\left(x_{i}\right) \in X^{\omega}: x_{i}=\alpha_{i} \forall i=1, \ldots, n\right\}$. Similarly to [PW] I consider a symbolic construction which is determined by a measurable family of closed (usually, compact) subsets $\Delta_{\alpha^{(n)}}^{\omega}$ of $\mathbb{R}^{d}$ (or of another good complete metric space) defined for all $\alpha \in X^{\omega}$ and such that: $\Delta_{\alpha^{(n)}}^{\omega} \cap \Delta_{\beta^{(n)}}^{\omega}=\emptyset$ if $\alpha^{(n)} \neq \beta^{(n)}, \Delta_{\alpha^{(n+1)}}^{\omega} \subset \Delta_{\alpha^{(n)}}^{\omega}$, 
and $\lim _{n \rightarrow \infty} \max _{\alpha^{(n)}} \operatorname{diam}\left(\Delta_{\alpha^{(n)}}^{\omega}\right)=0$. Now one is interested in dimension parameters of the limiting sets $K^{\omega}=\bigcap_{n=1}^{\infty} \bigcup_{\alpha^{(n)}: C_{\alpha(n)}^{\omega} \neq \emptyset} \Delta_{\alpha^{(n)}}^{\omega}$. Suppose that there exist random variables $K_{n}^{\omega}>0$ and $0<\lambda_{i}^{\omega}<1, \stackrel{\alpha}{i}=1, \ldots, m(\omega)$, such that each $\Delta_{\alpha(n)}^{\omega}$ contains a ball of radius $\left(K_{n}^{\omega}\right)^{-1} r_{\alpha^{(n)}}^{\omega}$ and is contained in a ball of radius $K_{n}^{\omega} r_{\alpha^{(n)}}^{\omega}$, where $r_{\alpha(n)}^{\omega}=\lambda_{\alpha_{n}}^{\theta^{n-1} \omega} \cdots \lambda_{\alpha_{2}}^{\theta \omega} \lambda_{\alpha_{1}}^{\omega}, \int \min _{i} \log \lambda_{i}^{\omega} d P(\omega)>-\infty$, and with probability one $\lim _{n \rightarrow \infty} \frac{1}{n} \log K_{n}^{\omega}=0$. Then similarly to Theorem 4.8 one can show that with probability one $H D\left(K^{\omega}\right)=t_{0}$ provided $Q^{(r)}\left(t_{0} \log \lambda\right)=0$, where $Q^{(r)}$ is the relativized topological pressure of the random subshift defined above and the function $\lambda$ on $X=\left\{(x, \omega): x \in X^{\omega}\right\}$ is given by $\lambda^{\omega}(x)=\lambda_{x_{1}}^{\omega}, x=\left(x_{i}\right) \in X^{\omega}$. If $\Delta_{\alpha^{(n)}}^{\omega}=f_{\alpha_{n}}\left(\theta^{n-1} \omega\right) \circ \cdots \circ f_{\alpha_{2}}(\theta \omega) \circ f_{\alpha_{1}}(\omega)(\Delta)$, where $f_{1}(\omega), \ldots, f_{m(\omega)}(\omega): \Delta \rightarrow \Delta$ are conformal contractions and $\Delta$ is a fixed compact set, then we arrive at a partial case of the above construction. If, in addition, the random subshift is of finite type, we obtain a certain random graph directed (or Markov) construction. It is possible to study also constructions governed by random sofic systems. Furthermore, similarly to Remark 4.13, under some additional assumptions I can estimate the Hausdorff dimension of subsets of points $z \in K^{\omega}$ such that their symbolic representations $\alpha=\alpha(z)=\left(\alpha_{1}, \alpha_{2}, \ldots\right) \in X^{\omega}, z=\bigcap_{\alpha^{(n)}} \Delta_{\alpha^{(n)}}^{\omega}$, have prescribed frequencies

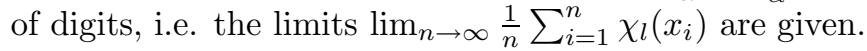

4.15. Remark. Employing $[\mathrm{ABD}]$ (see also $[\mathrm{Pe}]$ ), one can extend the notion of the correlation dimension to random transformations. Let $f(\omega): X \rightarrow X$ be continuous random transformations, $\omega \in \Omega$, and let $\theta: \Omega \rightarrow \Omega$ be a $P$-preserving ergodic transformation on $\Omega$. Let $\mu \in \mathcal{P}(X \times \Omega)$ be an ergodic invariant measure of the skew product transformation $\tau$ given by (1.8) with the marginal $P$ on $\Omega$, i.e. $d \mu(x, \omega)=d \mu^{\omega}(x) d P(\omega)$. Set

$$
C^{\omega}(x, n, r)=\frac{2}{n(n-1)} \operatorname{card}\left\{(i, j): \operatorname{dist}\left(f^{i}(\omega) x, f^{j}(\omega) x\right) \leq r \text { for } 0 \leq i<j<n\right\} .
$$

Then, with probability one, for $\mu^{\omega}$-a.a. $x$,

$$
\lim _{n \rightarrow \infty} C^{\omega}(x, n, r)=\int_{X \times X} \int_{\Omega \times \Omega} \chi_{\{\operatorname{dist}(y, z) \leq r\}} d \mu^{\omega}(x) d \mu^{\tilde{\omega}}(y) d P(\omega) d P(\tilde{\omega}),
$$

provided that, for $P \times P$-a.a. $(\omega, \tilde{\omega})$,

$$
\mu^{\omega} \times \mu^{\tilde{\omega}}\{(y, z): \operatorname{dist}(y, z)=r\}=0 .
$$

One can further describe sets of $r$ 's for which this condition holds true similarly to $[\mathrm{Pe}]$.

\section{REFERENCES}

[ABD] J. Aaronson, R. Burton, H. Dehling, D. Gilat, T. Hill, B. Weiss, Strong laws for L- and $U$-statistics, Trans. Amer. Math. Soc. (to appear).

[Bed] T. Bedford, On Weierstrass-like functions and random recurrent sets, Math. Proc. Camb. Phil. Soc. 106 (1989), 325-342. MR 91c:26010

[Be] A. S. Besicovitch, On the sum of digits of real numbers represented in the dyadic system, Math. Annalen 110 (1934), 321-330. 
[Bi1] P. Billingsley, Hausdorff dimension in probability theory. I, II, Illinois J. Math 4 (1960), 187-209; 5 (1961), 291-298. MR 24:A1750, MR 22:11094

[Bi2] P. Billingsley, Ergodic Theory and Information, John Wiley, New York, 1965. MR 33:254

[Bi3] P. Billingsley, Hausdorff dimension: self-similarity and independent processes; crosssimilarity and Markov processes, in: Statistics and Probability: A Raghu Raj Bahadur Festschrift (J. K. Ghosh, S. K. Mitra, K. R. Parthasarathy and B. L. S. Prakasa Rao, eds.), Wiley Eastern Ltd, 1993, pp. 97-134.

[Bo] T. Bogenschutz, Entropy, pressure, and a variational principle for random dynamical systems, Random \& Comp.Dyn. 1 (1992), 99-116. MR 93:28023

[BG1] T. Bogenschutz and V. M. Gundlach, Symbolic dynamics for expanding random dynamical systems, Random \& Comp.Dyn. 1 (1992), 219-227. MR 93j:58042

[BG2] T. Bogenschutz and V. M. Gundlach, Ruelle's transfer operator for random subshifts of finite type, Ergod. Th. \& Dyn. Sys. 15 (1995), 413-447. CMP 95:14

[BK] M. Brin and A. Katok, On local entropy, in: Geometric Dynamics, Lect. Notes in Math. 1007, Springer-Verlag, New York, 1983, pp. 30-38. MR 85c:58063

[Ca] H. Cajar, Billingsley Dimension in Probability Spaces, Lect. Notes in Math. 892, SpringerVerlag, Berlin, 1981. MR 84a:10055

[DZ] A. Dembo and O. Zeitouni, Large Deviations Techniques and Applications, Jones and Bartlett, Boston, 1993. MR 95a:60034

[Eg] H. G. Eggleston, The fractional dimension of a set defined by decimal properties, Quart. J. Math. Oxford Ser. 20 (1949), 31-36. MR 11:88

[Fa] K. Falconer, Fractal Geometry (Mathematical Foundations and Applications), J. Wiley \& Sons, Chichester, 1990. MR 92j:28008

[Fu] H. Furstenberg, Disjointness in ergodic theory, minimal sets, and a problem in Diophantine approximation, Math. Syst. Th. 1 (1967), 1-49. MR 35:4369

[Ki1] Y. Kifer, Large deviations in dynamical systems and stochastic processes, Trans. Amer. Math. Soc. 321 (1990), 505-524. MR 91e:60091

[Ki2] Y. Kifer, Equilibrium states for random expanding transformations, Random \& Comp.Dyn. 1 (1992), 1-31. MR 93j:58075

$[\mathrm{KK}] \mathrm{K}$. Khanin and Y. Kifer, Thermodynamic formalism for random transformations and statistical mechanics, Sinai's Moscow Seminar on Dynamical Systems (L. A. Buninovich, B. M. Gurevich, Ya. B. Pesin, eds.), AMS Translations-Series 2, 1995.

[KP1] R. Kenyon and Y. Peres, Intersecting random translates of invariant Cantor sets, Invent. Math. 104 (1991), 601-629. MR 92g:28018

[KP2] R. Kenyon and Y. Peres, Measures of full dimension on affine-invariant sets, Ergod. Th. \& Dynam. Sys. 15 (1995).

[LP] L. Lovász and M. D. Plummer, Matching Theory, North-Holland, Amsterdam, 1986. MR 88b:90087

[LW] F. Ledrappier and P. Walters, A relativized variational principle for continuous transformations, J. London Math. Soc. 16 (1977), 568-576. MR 57:16540

[Mc] C. McMullen, The Hausdorff dimension of general Sierpinski carpets, Nagoya Math. J. 96 (1984), 1-9. MR 86h:11061

[MO] A. W. Marshall and I. Olkin, Inequalities: Theory of Majorization and Its Applications, Academic Press, New York, 1979. MR 81b:00002

[Ol] L. Olsen, Random Geometrically Graph Directed Self-Similar Multifractals, Pitman Research Notes in Mathematics, vol. 307, Longman Sci. Tech., Harlow, 1994. MR 95j:28006

[Pe] Y. Pesin, On rigorous mathematical definitions of correlation dimension and generalized spectrum for dimensions, J. Stat. Phys. 71 (1993), 529-547. MR 94d:28008

[Pey] J. Peyrière, Calculs de dimensions de Hausdorff, Duke Math. J. 44 (1977), 591-601. MR 56:3257

[Ph] W. Philipp, Limit theorems for lacunary series and uniform distribution mod 1, Acta Arithm. 26 (1975), 241-251. MR 52:325

[PW] Y. Pesin and H. Weiss, On the dimension of deterministic and random Cantor-like sets, symbolic dynamics, and the Eckmann-Ruelle conjecture, Preprint (1994).

[Ru] D. Ruelle, Bowen's formula for the Hausdorff dimension of self-similar sets, in: Scaling and Self-similarity in Physics (Progress in Physics 7), Birkhäuser, Boston, 1983, pp. 351357. MR 85d:58051 
[Sm] M. Smorodinsky, Singular measures and Hausdorff measures, Israel J. Math. 7 (1969), 203-206. MR 40:3589

[St] V. Strassen, The existence of probability measures with given marginals, Ann. of Math. Stat. 36 (1965), 423-439. MR 31:1693

[Wa] P. Walters, Invariant measures and equilibrium states for some mappings which expand distances, Trans. Amer. Math. Soc. 236 (1978), 121-153. MR 57:6371

[Yo] L.-S. Young, Dimension, entropy, and Lyapunov exponents, Ergod. Th. \& Dyn. Sys. 2 (1982), 109-129. MR 84h:58087

Institute of Mathematics, Hebrew University of Jerusalem, Givat Ram, Jerusalem 91904, ISRAEL

E-mail address: kifer@math.huji.ac.il 